\title{
Gas expulsion in massive star clusters?
}

\section{Constraints from observations of young and gas-free objects}

\author{
Martin G. H. Krause ${ }^{1,2,3}$, Corinne Charbonnel ${ }^{4,5}$, Nate Bastian ${ }^{6}$, and Roland Diehl ${ }^{2,3}$ \\ ${ }^{1}$ Universitäts-Sternwarte München, Ludwig-Maximilians-Universität, Scheinerstr. 1, 81679 München, Germany \\ e-mail: krause@mpe.mpg.de \\ 2 Max-Planck-Institut für extraterrestrische Physik, Giessenbachstr. 1, 85741 Garching, Germany \\ 3 Excellence Cluster Universe, Technische Universität München, Boltzmannstrasse 2, 85748 Garching, Germany \\ ${ }^{4}$ Geneva Observatory, University of Geneva, 51 Chemin des Maillettes, 1290 Versoix, Switzerland \\ 5 IRAP, UMR 5277 CNRS and Université de Toulouse, 14 Av. E. Belin, 31400 Toulouse, France \\ 6 Astrophysics Research Institute, Liverpool John Moores University, 146 Brownlow Hill, Liverpool L3 5RF, UK
}

Received 6 June 2015 / Accepted 13 December 2015

\section{ABSTRACT}

\begin{abstract}
Context. Gas expulsion is a central concept in some of the models for multiple populations and the light-element anti-correlations in globular clusters. If the star formation efficiency was around 30 per cent and the gas expulsion happened on the crossing timescale, this process could preferentially expel stars born with the chemical composition of the proto-cluster gas, while stars with special composition born in the centre would remain bound. Recently, a sample of extragalactic, gas-free, young massive clusters has been identified that has the potential to test the conditions for gas expulsion.

Aims. We investigate the conditions required for residual gas expulsion on the crossing timescale. We consider a standard initial mass function and different models for the energy production in the cluster: metallicity-dependent stellar winds, radiation, supernovae and more energetic events, such as hypernovae, which are related to gamma ray bursts. The latter may be more energetic than supernovae by up to two orders of magnitude.

Methods. We computed a large number of thin-shell models for the gas dynamics, and calculated whether the Rayleigh-Taylor instability is able to disrupt the shell before it reaches the escape speed.

Results. We show that the success of gas expulsion depends on the compactness index of a star cluster $C_{5} \equiv\left(M_{*} / 10^{5} M_{\odot}\right) /\left(r_{\mathrm{h}} / \mathrm{pc}\right)$, with initial stellar mass $M_{*}$ and half-mass radius $r_{\mathrm{h}}$. For given $C_{5}$, a certain critical, local star formation efficiency is required to remove the rest of the gas. Common stellar feedback processes may not lead to gas expulsion with significant loss of stars above $C_{5} \approx 1$. Considering pulsar winds and hypernovae, the limit increases to $C_{5} \approx 30$. If successful, gas expulsion generally takes place on the crossing timescale.

Some observed young massive clusters have $1<C_{5}<10$ and are gas-free at $\approx 10$ Myr. This suggests that gas expulsion does not affect their stellar mass significantly, unless powerful pulsar winds and hypernovae are common in such objects.

By comparison to observations, we show that $C_{5}$ is a better predictor for the expression of multiple populations than stellar mass. The best separation between star clusters with and without multiple populations is achieved by a stellar winds-based gas expulsion model, where gas expulsion would occur exclusively in star clusters without multiple populations. Single and multiple population clusters also have little overlap in metallicity and age.

Conclusions. Globular clusters should initially have $C_{5} \lesssim 100$, if the gas expulsion paradigm was correct. Early gas expulsion, which is suggested by the young massive cluster observations, hence would require special circumstances, and is excluded for several objects. Most likely, the stellar masses did not change significantly at the removal of the primordial gas. Instead, the predictive power of the $C_{5}$ index for the expression of multiple populations is consistent with the idea that gas expulsion may prevent the expression of multiple populations. On this basis, compact young massive clusters should also have multiple populations.
\end{abstract}

Key words. ISM: bubbles - globular clusters: general - galaxies: star clusters: general

\section{Introduction}

The formation of stars is associated with radiation, stellar winds, and energetic explosions. This feedback is expected to easily clear away surrounding gas (e.g. Freyer et al. 2003; Acreman et al. 2012; Fierlinger et al. 2012; Krause et al. 2013b; Krumholz et al. 2014). Indeed, star-forming regions are observed to be actively clearing away gas at an age of about $10^{6}$ years (e.g. Voss et al. 2010, 2012; Preibisch et al. 2012; Galván-Madrid et al. 2013; Klaassen et al. 2014), and to be free of dense gas from about a few Myr after the onset of star formation (e.g., the review by Lada \& Lada 2003).

The gravitational binding energy of a star-forming region increases with the square of the total mass, however, whereas feedback processes are directly proportional to the latter. A certain mass limit is therefore expected, above which the released energy is insufficient to remove the remaining gas (compare, e.g., Decressin et al. 2010; Krause et al. 2012). Candidate regions for this to occur certainly include nuclear star clusters in galaxies, where a super-massive black hole typically adds to the gravity of the stars (e.g. Schartmann et al. 2010). But other star clusters, in particular globular clusters (GCs), can also be so massive and compact that the gravitational binding energy of the gas becomes important.

If at the time of gas clearance the gravitational potential of such clusters is dominated by this gas, and if the gas clearance happens sufficiently fast, comparable to the crossing timescale, then many stars if not all (disruption) are also lost as a result 
of the drastic change in gravitational potential (e.g., Bastian \& Goodwin 2006; Goodwin \& Bastian 2006; Bastian et al. 2008; Gieles \& Bastian 2008; Baumgardt et al. 2008; Decressin et al. 2010; Marks et al. 2012; Pfalzner \& Kaczmarek 2013; Pfalzner et al. 2014; Banerjee \& Kroupa 2016). This is commonly referred to as gas expulsion (Portegies Zwart et al. 2010; Banerjee \& Kroupa 2015, for reviews).

Gas expulsion is central in explanations for the chemical peculiarities in GCs and is invoked in certain scenarios as a possibility to eject a large number of less tightly bound first population stars born with the pristine composition of the proto-cluster gas. At the opposite, second population stars showing peculiar abundance properties (e.g. the $\mathrm{O}-\mathrm{Na}$ anti-correlation) and born in the cluster centre would remain bound after gas expulsion (D'Ercole et al. 2008, 2010; Krause et al. 2013a). This is one possibility to solve the so-called mass-budget problem to explain the number distribution of stars along the O-Na anti-correlation. Other possibilities exist though, that call for a modified initial mass function (IMF; Prantzos \& Charbonnel 2006; Charbonnel et al. 2014). The stars ejected from GCs might form a substantial population of galactic halos (e.g., Schaerer \& Charbonnel 2011; Larsen et al. 2014b).

Young massive clusters (YMCs) are important test cases for the gas expulsion idea because the involved timescales, masses, and energetics may be constrained directly by observations (e.g., Portegies Zwart et al. 2010). Some extragalactic objects appear to be gas-free after 10 Myr or less (Bastian et al. 2014; Hollyhead et al. 2015) and are thus very interesting objects for comparison to theoretical expectations.

We have investigated gas expulsion in the context of models that aim to explain the light-element anti-correlations in GCs with massive progenitors (Krause et al. 2012, Paper I). We found that gas expulsion would not work for common cluster parameters because the outflow would be disrupted by instabilities before escape speed was reached. An unusually strong power source, like the coherent onset of Eddington-strength accretion on all neutron stars and stellar black holes in a cluster, would be required to still allow for gas expulsion. In this case, however, gas expulsion would occur relatively late in the cluster life, at the end of the turbulent $\mathrm{SNe}$ phase (i.e. typically at ages of about $40 \mathrm{Myr}$ ), which is incompatible with the fact that YMCs appear to be gas-free after $\approx 4$ Myr.

We place our modelling in a more general context here. We first review constraints on gas expulsion from observations of stellar kinematics, light-element anti-correlations, the population of halo stars, and gas (or the absence thereof) in YMCs in Sect. 2. We describe our method in Sect. 3), and present the results in Sect. 4. We present detailed gas expulsion models for a sample of YMCs from Bastian et al. (2014) in Sect. 4.1. In Sect. 4.2 we show a grid of calculations for various feedback scenarios. We discuss the results in Sect. 5 and summarise the conclusions in Sect. 6.

\section{Constraints on gas expulsion}

\subsection{Star cluster mass functions and kinematics}

Lada \& Lada (2003) found that "the embedded cluster birthrate exceeds that of visible open clusters by an order of magnitude or more". They concluded that many clusters were dissolved at an early age, consistent with gas expulsion ideas. Bressert et al. (2010) then determined the distribution of the densities of young stellar objects in the solar neighbourhood, and found a continuous distribution around $22 \mathrm{stars}_{\mathrm{pc}} \mathrm{p}^{-2}$. They concluded that the number of embedded star clusters strongly depends on the adopted star density threshold, complicating conclusions about early star cluster dissolution. They also found, however, that class I (younger) objects have, on average, larger densities than class II (older) objects, supporting the idea that star-forming regions often dissolve. The environment has a strong effect on the evolution of the cluster mass functions (see the review by Longmore et al. 2014). It is therefore difficult to constrain gas expulsion in this way.

Young ( $\approx 10 \mathrm{Myr}$ ), exposed star clusters often appear to have supervirial velocity dispersions (Gieles et al. 2010). This has been discussed as evidence for dissolution after gas expulsion (e.g., Goodwin \& Bastian 2006). It is, however, expected that many clusters would have re-virialized by the time of observation (Baumgardt \& Kroupa 2007; Gieles et al. 2010; Portegies Zwart et al. 2010). An interpretation in terms of a large contribution from binaries to the velocity dispersion (compare, e.g., Leigh et al. 2015; Oh et al. 2015) seems more probable (Gieles et al. 2010; Rochau et al. 2010; Cottaar et al. 2012; Clarkson et al. 2012; Hénault-Brunet et al. 2012; Cottaar \& Hénault-Brunet 2014). Therefore, it is also difficult to constrain gas expulsion in this way.

\subsection{Light-element anti-correlations in globular clusters}

With the former tailwind from the general research on star clusters, gas expulsion assumed a central role for the understanding of GCs (e.g., Goodwin 1997a,b; Fenner et al. 2004; Prantzos \& Charbonnel 2006; Parmentier \& Gilmore 2007; Baumgardt et al. 2008; D'Ercole et al. 2008; Decressin et al. 2010; Marks \& Kroupa 2010; Vesperini et al. 2010; Schaerer \& Charbonnel 2011; Marks et al. 2012). In contrast to the vast majority of open (lower mass) clusters studied so far, almost all of the GCs show chemical peculiarities, most prominently the light-element anticorrelations (Charbonnel 2010; Gratton et al. 2012, for reviews), which may well point to fundamental differences in the mode of star formation.

The light-element anti-correlations in GC stars, most prominently between $\mathrm{Na}$ and $\mathrm{O}$, together with the constancy of other elements such as Fe imply that hydrogen-burning products from more massive stars than those currently present have been reprocessed, possibly mixed with remaining gas from the star formation event to form the currently observed low-mass stars (Gratton et al. 2001, 2012; Prantzos \& Charbonnel 2006; Prantzos et al. 2007; Charbonnel 2010). O-rich and Na-poor stars form the first population, whereas the O-poor and $\mathrm{Na}$ rich stars constitute the second one. Intermediate-mass asymptotic giant branch $(\mathrm{AGB})$ stars $\left(\approx 6 M_{\odot}-11 M_{\odot}\right.$ Ventura et al. 2001, 2013; Ventura \& D'Antona 2009, 2011; D'Ercole et al. 2010) and fast-rotating massive stars (FRMS; $\gtrsim 25 M_{\odot}$ Maeder \& Meynet 2006; Prantzos \& Charbonnel 2006; Decressin et al. 2007; Krause et al. 2013a; Charbonnel et al. 2014), variants and combinations of these object classes (de Mink et al. 2009; Sills \& Glebbeek 2010; Bastian et al. 2013; Cassisi \& Salaris 2014), and recently also supermassive stars (Denissenkov \& Hartwick 2014) have received attention as possible sources of the enriched material. From the nucleosynthesis point of view, FRMS, or massive stars in binary systems (de Mink et al. 2009), are perhaps the most obvious polluter candidates: they produce the $\mathrm{Na}-\mathrm{O}$ anti-correlation directly (Decressin et al. 2007), as opposed to AGB stars, in which the direct correlation in their ejecta has to be turned into an anti-correlation by a precisely prescribed mixing procedure (Ventura et al. 2013). 
Because the ejecta mass is low in both, the AGB and the FRMS scenario, gas expulsion has been identified as a possibility to enhance the ratio of second- to first-generation stars (mass budget problem, D'Ercole et al. 2008, 2011; Cabrera-Ziri et al. 2015): If the second generation was formed close to the massive stars in a mass-segregated star cluster, the less tightly bound first generation would have been lost preferentially (Decressin et al. 2010).

For these models to work, i.e. remove $\gtrsim 95$ percent of the stars, gas expulsion needs to be explosive (Decressin et al. 2010; Khalaj \& Baumgardt 2015), i.e. happen on the crossing timescale of the cluster. According to our models (Krause et al. 2012, 2013a), stellar winds and supernovae do not provide enough power for this process. Energy release from the combined accretion on to the dark remnants of the massive stars (neutron stars and black holes) might perhaps accomplish the task. This would then, however, happen late, about 35 Myr after cluster formation, when the massive stars would have turned into such dark remnants.

If gas expulsion would work in multiple population clusters, one would expect a correlation between the total stellar mass and the fraction of enriched stars, which is, however, not seen (Khalaj \& Baumgardt 2015; Bastian \& Lardo 2015).

\subsection{Extragalactic globular clusters and stars in galactic haloes}

Extragalactic globular clusters (XGCs) are very similar to GCs in age and metallicity (Brodie \& Strader 2006, for a review), although there are also some differences (e.g., Montes et al. 2014). Associations of XGCs with tidal streams suggest that the majority of globular clusters was formed in dwarf galaxies and accreted with them to the haloes of larger galaxies (Mackey et al. 2010, 2013, 2014; Huxor et al. 2014; Veljanoski et al. 2014).

Old GCs in the Large Magellanic Cloud have been shown to exhibit the O-Na anti-correlation (Mucciarelli et al. 2009). From integrated spectra of nearby dwarf galaxies, Larsen et al. (2014a,b) found evidence for $\mathrm{Na}$ spreads, consistent with the presence of the Na-O anti-correlation and other GC characteristics. Comparing the masses of XGCs to the total mass of halo stars with the same metallicity in the respective host galaxies, the latter authors constrained the ejection of first-generation lowmass stars to four times the current cluster mass, a much tighter constraint than from the analogous argument for the Milky Way (Schaerer \& Charbonnel 2011). This number has to accommodate secular effects as well as star clusters that have been disrupted completely. Unless stars ejected from the GCs in Fornax also had enough kinetic energy to disappear from the parent dark-matter halo, this places tight constraints on gas expulsion.

\subsection{Young massive star clusters}

YMCs (Portegies Zwart et al. 2010; Longmore et al. 2014, for reviews) provide quite possibly very interesting complementary insight into what occurred in the infancy of GCs. They reach masses of up to $10^{8} M_{\odot}$, which is comparable to the initial mass postulated for GCs from self-enrichment considerations, and have core radii of one to a few parsec, similar to GCs. A possible difference concerns the metallicity, which reaches down to lower values in the case of GCs. Some GCs, however, show the anti-correlation, but have higher metallicities than some YMCs. Metallicity affects the thermodynamics of the gas, which may have a rather strong effect on the star formation process (e.g.,
Peters et al. 2012). It has not yet been possible to determine, whether an $\mathrm{Na}-\mathrm{O}$ anti-correlation such as characteristic for $\mathrm{GCs}$ is present in YMCs.

Star formation and gas has been searched for in YMCs by multiwavelength studies including $\mathrm{H} \alpha$ and infrared (Whitmore et al. 2011; Bastian et al. 2014; Hollyhead et al. 2015). Embedded clusters are routinely detected. Whitmore et al. (2011) and Hollyhead et al. (2015) demonstrate for a large sample that in massive clusters up to $\approx 10^{5} M_{\odot}$ there is no gas present after about 4 Myr. Bastian et al. (2014) show for a small sample around $10^{6} M_{\odot}$ and ages from 4 to $15 \mathrm{Myr}$ that gas is not present, entirely consistent with the studies at lower masses. Thus, around 4 Myr the gas has either been expelled, shed more gently in a mass-loaded wind, or it has been very efficiently transformed into stars.

\section{Methods}

\subsection{How may gas disappear from a star cluster?}

In general, the remaining gas from which the stars in a cluster formed may be cleared in three ways: first, star formation can proceed and use up the gas. Second, a steady, moderately massloaded wind (e.g., Palouš et al. 2013; Calura et al. 2015) may remove gas over timescales that are long compared to the crossing time, which is probably what occurs in galactic winds (e.g., Strickland \& Heckman 2009; von Glasow et al. 2013). In this case, the change in gravitational potential is comparatively slow, such that the effect on the stellar kinematics is moderate, and the fraction of stars lost is small (Baumgardt \& Kroupa 2007). This would not be of interest in the present context. Finally, superbubble formation is a third possibility (Krause et al. 2012, Paper I in the following). Here, the hot gas is located inside a thin shell, such that the majority of the gas in the thin shell may be efficiently accelerated. This is the most plausible setup for gas expulsion on the crossing timescale and thus has the potential for strong effects on the stellar kinematics.

The gas expulsion paradigm involves ejection of a gas mass comparable to the total mass on the crossing timescale $\left(\tau_{\mathrm{c}}=\right.$ $0.2 \mathrm{Myr} r_{\mathrm{h}, 3}^{3 / 2} M_{\mathrm{tot}, 6}^{-1 / 2}, r_{\mathrm{h}, 3}$ : half-mass radius in units of $3 \mathrm{pc}, M_{\mathrm{tot}, 6}$ : total cluster mass in units of $\left.10^{6} M_{\odot}\right)$. The binding energy due to the self-gravity of the gas is $10^{52} \mathrm{erg}\left(1-\epsilon_{\mathrm{SF}}\right) M_{\mathrm{tot}, 6}^{2} r_{\mathrm{h}, 3}^{-1}$, where we have used the formula for a Plummer star cluster model (Baumgardt et al. 2008). $\epsilon_{\mathrm{SF}}$ is the star formation efficiency. Here, we mean a local star formation efficiency, namely the initial stellar mass of a cluster divided by the total baryonic mass (stars and gas) within the space occupied by the forming stellar population of a given star cluster. The binding energy thus approaches the energy that supernovae can produce within a crossing time (compare Decressin et al. 2010). To still expel the gas in these circumstances, a very efficient gas expulsion mechanism is required. A superbubble is therefore a promising candidate to fulfil the constraints.

\subsection{Energy production}

Stellar winds, ionising radiation, and radiation pressure from massive stars appear very early in the formation of a star cluster (compare, e.g., Dale et al. 2015). After a few Myr, stellar explosions comprise the main energy source, and after a given star has exploded, it may release energy by accretion, either from a companion star or, for favourable conditions, from the intracluster medium. Technically, we derive the energy production rate 
by integrating the stellar sources over the IMF, similar to, e.g., Shull \& Saken (1995). In a solar-metallicity star cluster, winds and supernovae combine to maintain a similar energy input rate over tens of Myr (Voss et al. 2009).

We consider the following cases for the energy production:

1. Stellar winds with the same energy injection rates as in Paper I, but scaled for metallicity $Z$ as $Z^{0.7}$ (Maeder \& Meynet 2012), according to the metallicity of each cluster. We show in Appendix A that the gas pressure due to thermalised stellar winds is much higher than the radiation pressure and that radiation pressure alone may hardly ever lead to gas expulsion in massive star clusters. Therefore, we neglect the radiation pressure. Effects of ionisation are also negligible in the present context because the escape speed in massive star clusters (compare below) is much higher than the typical sound speed in photo-ionised gas (compare, e.g., Dale et al. 2014).

2. Supernovae explosions of the massive stars at the end of their evolution. We assume that all massive stars explode as $\mathrm{SNe}$, although this is subject to caution, because stars more massive than $\approx 25 M_{\odot}$ might in fact silently turn into black holes. For the standard case, we assume an energy of $10^{51}$ erg per explosion. Such explosions may be enhanced by rapid spin-down, however, if a fast rotating dark remnant was produced ("hypernova", e.g., Langer 2012). The energy that may be released in this way is limited by the extractable energy of black holes of mass $M_{\mathrm{DM}}$, (Christodoulou 1970; Hawking 1972; Penrose 1972; Blandford \& Znajek 1977), $E_{\mathrm{x}}=0.29 M_{\mathrm{DM}} c^{2}=5 \times 10^{54} \mathrm{erg} M_{\mathrm{DM}} /\left(10 M_{\odot}\right)$. Neutron stars will in general have lower rotational energies than this. Constraints from the spin-down power of observed young pulsars, $\gtrsim 10^{38} \mathrm{erg} \mathrm{s}^{-1}$ for $\gtrsim 10^{3}$ years (Kuiper \& Hermsen 2015 ), provide a lower limit to the potential total energy release of $3 \times 10^{48} \mathrm{erg}$. Observational constraints for processes related to rotating black holes may be taken from measurements of type Ic supernovae associated with GRBs (e.g., Mazzali et al. 2014), which are around $10^{52} \mathrm{erg}$. Heesen et al. (2015) found $\gtrsim 10^{52} \mathrm{erg}$ in a superbubble around the $>23 M_{\odot}$ black hole IC10-X1, plausibly related to a hypernova at its formation. If the strong explosions would be connected to a particular range of stellar masses, they might occur in a small time interval. In the context of gas expulsion, only the energy produced in a crossing time matters. Hence, it would be possible that all the strong explosions in a given cluster add up coherently to enable the gas expulsion process. We consider hypernovae with, individually, 10 and 100 times the standard explosion energy of $10^{51} \mathrm{erg}$. For a given simulation, all explosions have the same energy.

\subsection{Implementation}

We evolved the dynamical equations for the expansion of a spherically symmetric thin shell under conservation of energy and momentum,

$\frac{\partial}{\partial t}(\mathcal{M} v)=p A-\mathcal{M} g$

where, $\mathcal{M}=4 \pi \int_{0}^{r} \rho_{\mathrm{g}}\left(r^{\prime}\right) r^{\prime 2} \mathrm{~d} r^{\prime}$ is the mass in the shell, with the gas density $\rho_{\mathrm{g}}$. Shell radius and velocity are respectively denoted by $r$ and $v$. The shell's surface area is given by $A=4 \pi r^{2}$, and $g$ is the gravitational acceleration. The bubble pressure is $p=$ $(\gamma-1)\left(\eta E(t)-\mathcal{M} v^{2} / 2\right) / V$, with the bubble volume $V=4 \pi r^{3} / 3$, the energy injection law $E(t)$, an efficiency parameter $\eta$, and the ratio of specific heats, $\gamma=5 / 3$. We assume $p$ to dominate over the ambient pressure. In this approach, following essentially Brown et al. (1995), the gravity of the stars as well as the selfgravity of the gas are taken into account. Detailed 3D hydrodynamics simulations, which take into account heating and cooling processes in the gas and model instabilities in detail yield a timeaveraged radiative dissipation in supershells of about 90 per cent of the injected energy (Krause et al. 2013b; Krause \& Diehl 2014). We therefore conservatively assumed that 80 per cent of the injected energy is radiated and 20 per cent (our parameter $\eta$ ) is used to move the gas. We first assumed a star formation efficiency $\epsilon_{\mathrm{SF}}$ of 30 per cent for some standard runs because this is a reference value in gas expulsion studies (e.g., Decressin et al. 2010), but varied this parameter frequently as indicated in the individual investigations. We used the IMF from Kroupa et al. (2013). The initial condition is assumed to be a Plummer sphere for stars and gas.

Because gravity is strongest at the half-mass radius, the general form of the solution is a comparatively slow push across the half-mass radius, and then an acceleration thanks to the decreasing gravitational force. Unless the shell's deceleration exceeds gravity, it is prone to the Rayleigh-Taylor instability. We calculate analytically when modes comparable to the shell size have had enough time to grow. The Rayleigh-Taylor length scale $\lambda$ is given by $\lambda=(a-g) \tau^{2}$, where $a$ is the shell's acceleration, and $\tau$ the time interval for which the instability criterion, $a-g>0$, was fulfilled (compare Paper I). Note that a shell is always unstable if $a>0$ (shell acceleration), because gravity is attractive $(g<0)$. It is also unstable if the shell decelerates, but the magnitude of gravity exceeds the deceleration $(|g|>|a|)$. Stability requires deceleration and small gravity $(|g|<|a|)$.

When $\lambda$ exceeds the shell radius, the flow is expected to change character from a superbubble into a convective flow. This is a reasonable assumption: The timescale $\tau$ defined above is very similar to the growth time for a mixing layer (Poujade \& Peybernes 2010), and also simply the time a piece of shell material would need to reach the centre, if gravity dominates. Simulations show directly that smaller scale modes, for example ones comparable to the shell thickness, lead to minor diffusion of shell material into the bubble interior and bulk acceleration of the possibly fragmented shell (e.g., Krause 2005; Krause et al. 2013b), whereas modes comparable to the bubble size lead to large-scale overturn (e.g., Janka \& Mueller 1996; Hardcastle \& Krause 2013).

Any remaining mass loss is then considerably reduced. We refer to Paper I for more details about the numerics and basic behaviour of the solution.

\section{Results}

\subsection{Young massive clusters}

We first present results for the sample of YMCs recently compiled by Bastian et al. (2014) for the purpose of demonstrating the absence of gas in massive star clusters of ages younger than or just about 10 Myr. For the calculations, we adopted the masses and metallicities given in Bastian et al. (2014) (Table 1). We calculated the half-mass radius from the half-light radii given in Bastian et al. (2014) by multiplying by 1.7 which would be correct if the clusters were all Plummer spheres. 
M. G. H. Krause et al.: Gas expulsion in massive star clusters?

Table 1. Observational data and results of gas expulsion simulations for the YMC sample of Bastian et al. (2014).

\begin{tabular}{llccccccccc}
\hline \hline Galaxy & Cluster & $\begin{array}{c}\mathrm{Age}^{a} \\
(\mathrm{Myr})\end{array}$ & $\begin{array}{c}M_{*}{ }^{b} \\
\left(10^{5} M_{\odot}\right)\end{array}$ & $\begin{array}{c}r_{\mathrm{h}}{ }^{c} \\
(\mathrm{pc})\end{array}$ & $C_{5}{ }^{d}$ & $\begin{array}{c}Z^{e} \\
\left(Z_{\odot}\right)\end{array}$ & $\begin{array}{c}\mathrm{Ex} / \\
\mathrm{W}^{f}\end{array}$ & $\begin{array}{c}\mathrm{Ex} / \\
\mathrm{SN}^{f}\end{array}$ & $\begin{array}{c}\epsilon_{\mathrm{SF}, \mathrm{W}, \mathrm{c}}{ }^{g} \\
(\%)\end{array}$ & $\begin{array}{c}t_{\mathrm{ex}, \mathrm{w}}{ }^{h} \\
(\mathrm{Myr})\end{array}$ \\
\hline NGC 6946 & 1447 & $12 \pm 2.5$ & 8 & 17.4 & 0.46 & 0.5 & $\mathrm{Y}$ & $\mathrm{Y}$ & 20 & 0.63 \\
NGC 1569 & $\mathrm{A}$ & $6 \pm 1$ & 7.6 & 1.5 & 5.1 & 0.4 & $\mathrm{~N}$ & $\mathrm{~N}$ & 80 & 0.12 \\
& $\mathrm{~B}$ & $15 \pm 5$ & 14 & 2.4 & 5.9 & 0.4 & $\mathrm{~N}$ & $\mathrm{~N}$ & 80 & 0.13 \\
NGC 1705 & 1 & $12.5 \pm 2.5$ & 11 & 1.5 & 7.3 & 0.33 & $\mathrm{~N}$ & $\mathrm{~N}$ & 80 & 0.12 \\
NGC 1140 & 1 & $5 \pm 1$ & 11 & 14 & 0.79 & 0.5 & $\mathrm{Y}$ & $\mathrm{Y}$ & 30 & 0.48 \\
The Antennae & T352/W38220 & $4 \pm 2$ & 9.2 & 4.1 & 2.2 & 1 & $\mathrm{~N}$ & $\mathrm{~N}$ & 40 & 0.20 \\
& Knot S & $5 \pm 1$ & 16 & 14 & 1.1 & 1 & $\mathrm{~N}$ & $\mathrm{Y}$ & 40 & 0.52 \\
ESO 338-IG04 & Cluster 23 & $6_{-2}^{+4}$ & 50 & 8.9 & 5.6 & 0.2 & $\mathrm{~N}$ & $\mathrm{~N}$ & 80 & 0.22 \\
\hline
\end{tabular}

Notes. ${ }^{(a)}$ Age in Myr. ${ }^{(b)}$ Fiducial stellar mass, adopting the photometric mass estimate from Bastian et al. (2014). ${ }^{(c)} r_{\mathrm{h}}$ : half-mass radius, calculated from the half-light radius assuming a Plummer sphere. ${ }^{(d)}$ Compactness index $C_{5}=\left(M_{*} / 10^{5} M_{\odot}\right)\left(r_{\mathrm{h}} / \mathrm{pc}\right)^{-1}$. ${ }^{(e)}$ Metallicity. ${ }^{(f)}$ Success of gas expulsion assuming 30 per cent star formation efficiency by, respectively, stellar winds $(\mathrm{Ex} / \mathrm{W})$ and supernovae $(\mathrm{Ex} / \mathrm{SN})$. Y: yes, N: no. (g) Critical star formation efficiency for gas expulsion in the metallicity dependent massive-star wind scenario. With this efficiency, a supershell would just not yet escape. The simulation at a star formation efficiency ten percentiles higher then features successful gas expulsion. ${ }^{h}$ Time since the coeval starburst at which an eventually escaping shell crosses the half-mass radius, for the stellar wind case with $\epsilon_{\mathrm{SF}, \mathrm{W}, \mathrm{c}}$.

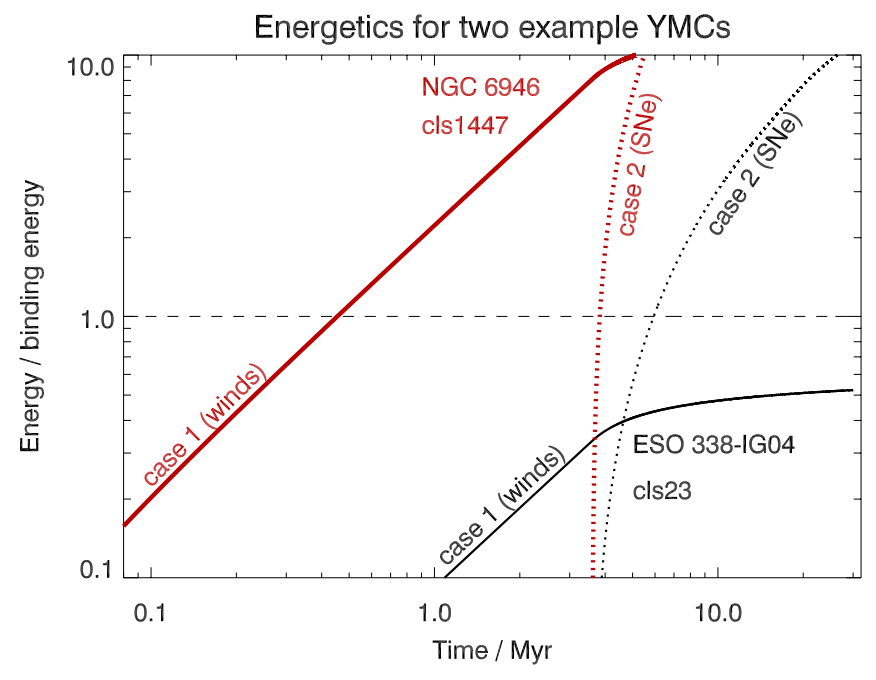

Fig. 1. Produced energy in units of the binding energy for a model with $\epsilon_{\mathrm{sf}}=0.3$ for two example clusters, ESO 338-IG04 cluster 23 (thinner black lines) and NGC 6946 cluster 1447 (thicker red lines), for different assumptions about the energy production: case 1 assumes stellar winds (solid lines); case 2 is for standard supernovae (dotted lines). The dashed black horizontal line indicates the binding energy.

\subsubsection{Cluster 1447 in the galaxy NGC 6946: gas expulsion for standard conditions}

As an example for a star cluster where gas expulsion would be feasible, we discuss the case of cluster 1447 in the galaxy NGC 6946. It has a stellar mass of $8 \times 10^{5} M_{\odot}$ and a comparatively large half-mass radius of $17 \mathrm{pc}$. It is gas-free at an age of 12 Myr. The energy tracks are shown in Fig. 1 (red lines). Stellar winds and supernovae are able individually to provide an energy comparable to the binding energy within less than a Myr.

We show the shell kinematics plot in Fig. 2. For both cases (winds left, supernovae right), the Rayleigh-Taylor scale stays comfortably below the shell radius at all times of interest (top diagrams). This means that only small scale Rayleigh-Taylor modes disturb the shell, but overall the shell is preserved. Only for short time intervals around the peak of the gravitational pull (bottom diagrams), the shell velocity drops below the local escape speed (middle diagrams). But since gravity and shell acceleration are too small to make the shell entirely unstable, the shell may reaccelerate as a whole, beyond escape speed. After crossing the half-mass radius, the gravitational pull declines, and the shell consequently accelerates (bottom diagrams). The shell would then continue to accelerate indefinitely, because of the strong decline of the density in the Plummer potential (compare Paper I). In reality it would soon be slowed down again by interaction with the ambient medium.

Therefore in that peculiar case, our model with standard assumptions for the SFE and the sources of energy predicts a successful gas expulsion, consistent with observations.

\subsubsection{Cluster 23 in the galaxy ESO 338-IG04: no gas expulsion for standard conditions}

Cluster 23 in ESO 338-IG04 has a mass of about $5 \times 10^{6} M_{\odot}$ and a half-mass radius of $9 \mathrm{pc}$. We again assumed a star formation efficiency of $\epsilon_{\mathrm{SF}}=0.3$.

The evolution of the energy production in this cluster is also shown in Fig. 1 (black lines). Stellar winds alone do not reach the binding energy for this cluster. For supernovae, the binding energy is reached only after about 1 Myr of supernova activity.

The energetics are reflected in the shell kinematics plots shown in Fig. 3. As expected, stellar winds (left plot) alone cannot expel the gas in this YMC for the adopted star formation efficiency. This is also evident from the fact that the shell velocity is unable to reach escape speed for the entire wind phase, up to about 3.5 Myr (middle diagram). The shell is pushed so gently across the half-mass radius that the Rayleigh-Taylor scale quickly exceeds the shell radius (top diagram). The shell is then destroyed by the instability, and most of the gas is expected to fall back (fountain flow). This is similar to the interstellar medium in disc galaxies (compare, e.g., von Glasow et al. 2013). The supershells studied here have a quite standard early deceleration phase (green line, bottom diagram). The deceleration, however, very quickly drops below gravity (red dashed line, bottom diagram). Consequently, it cannot stabilise the shell.

Supernovae (Fig. 3, right) could accelerate the supershell to escape speed within about $2 \mathrm{Myr}$ (5.4 Myr after the assumed coeval starburst middle diagram). However, this solution is also Rayleigh-Taylor unstable because the shell deceleration still drops too quickly below the gravitational acceleration. Gravity then boosts the Rayleigh-Taylor scale. It exceeds the shell radius from about $0.2 \mathrm{Myr}$ after onset of the supernovae. 

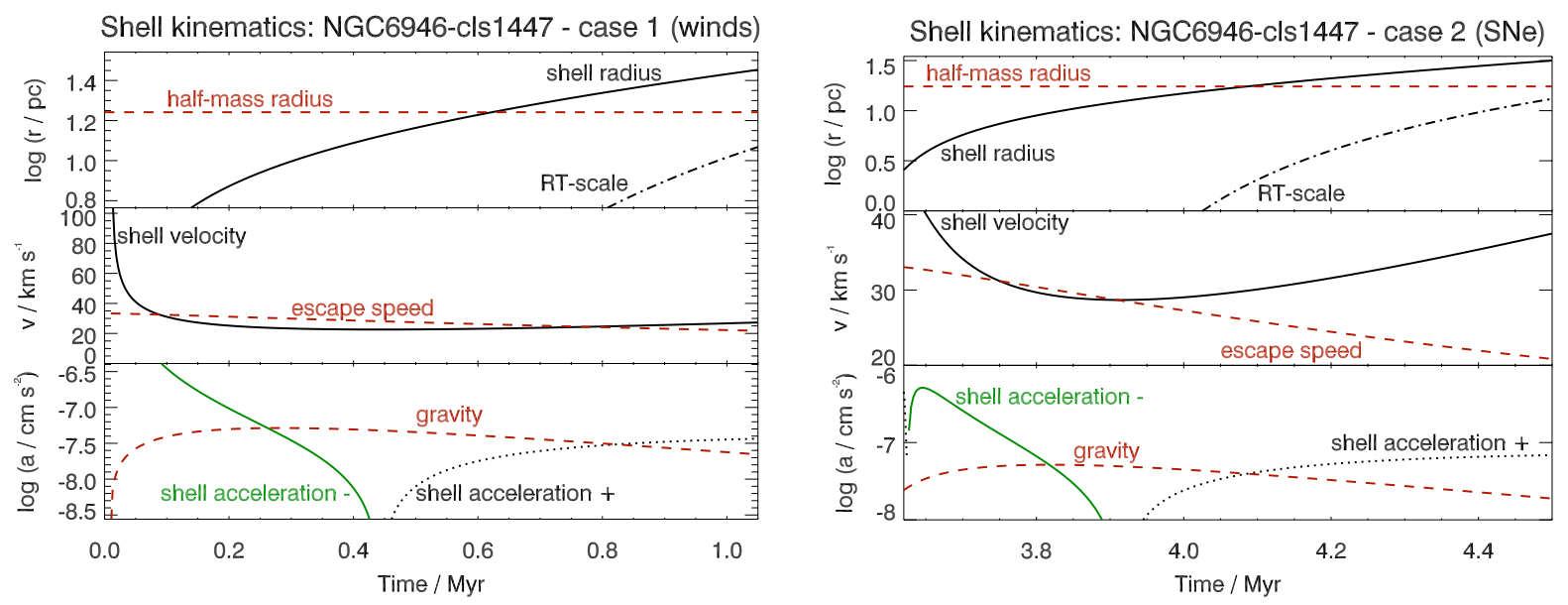

Fig. 2. Supershell kinematics for cluster 1447 in the galaxy NGC 6946. Left: case 1, stellar winds. Right: case 2, supernovae. The star formation efficiency is assumed to be 30 per cent in both cases. The timescale for the global evolution of the GC is chosen at the birth of a coeval first generation of stars. Within each kinematics plot, the upper diagram shows the bubble radius (solid black line) and the Rayleigh-Taylor scale (dash-dotted black line), with the red dashed line indicating the half-mass radius. The middle diagram displays the shell velocity (solid line) and the escape velocity at the current bubble radius (red dashed line). The acceleration is shown in the bottom diagram (positive: dotted black line, negative: solid green line), with the gravitational acceleration at the current radius shown as a red dashed line. Gas expulsion succeeds in both cases. Details in Sect. 4.1.1.
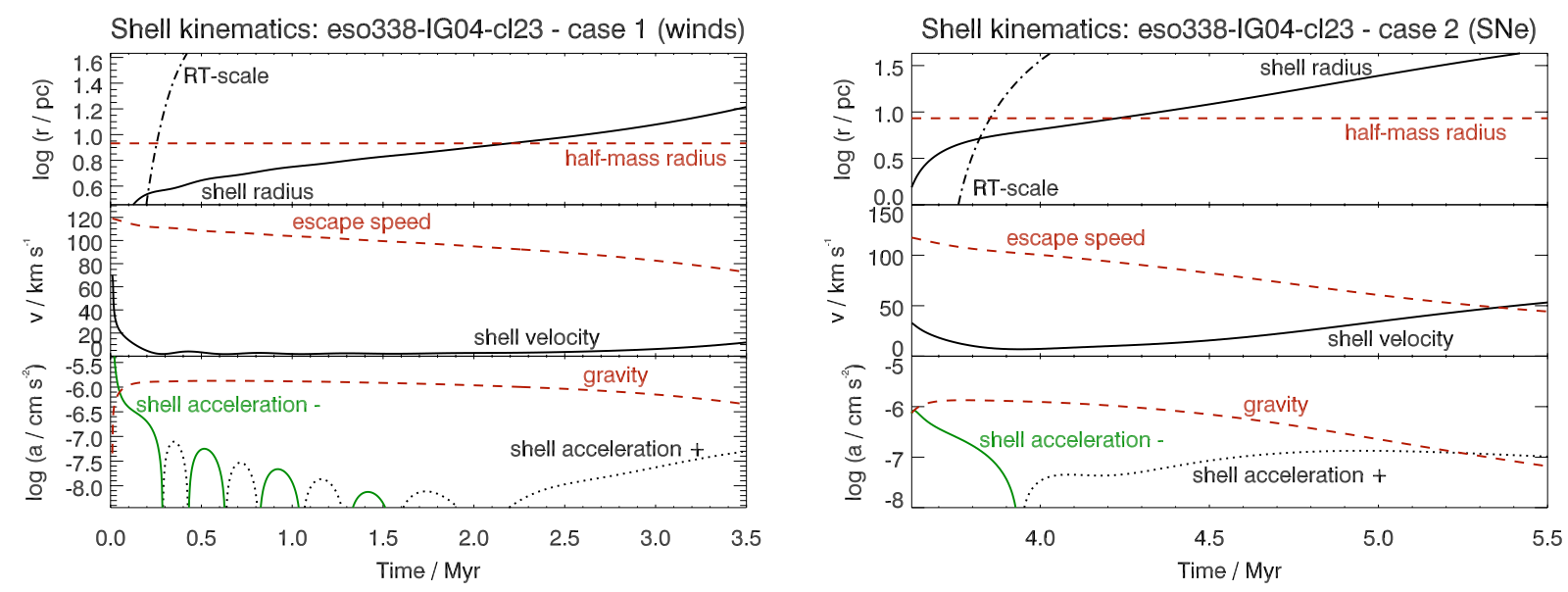

Fig. 3. Same as Fig. 2, but for cluster 23 in the galaxy ESO338-IG04. Gas expulsion does not succeed here. Details in Sect. 4.1.2.

Again, the shell is destroyed. Similar to the wind phase, the intracluster medium will then be dominated by blowout of individual bubbles (Tenorio-Tagle et al. 2015).

Therefore, if the star formation efficiency had been indeed 30 per cent, our calculations would predict that the unused gas could not have been expelled within the first few Myr by stellar winds, or standard supernovae. The ISM in this star cluster should then be convective, similar to the ISM of the Milky Way and other star-forming galaxies, where the stellar (and other) energy sources lead to turbulence with typical velocities of the order of $10 \mathrm{~km} \mathrm{~s}^{-1}$. Because this is small compared to the escape velocity, the gas would remain bound. However, the cluster is observed to be gas-free at an estimated age between 4 and 10 Myr.

\subsubsection{Other YMCs}

We have performed similar calculations for all star clusters of the sample of Bastian et al. (2014). The results are summarised in Table 1. For two of the objects, gas expulsion is predicted to be possible with a star formation efficiency of 30 per cent for both energy injection cases studied (winds and supernovae). For one cluster, stellar winds would have been insufficient, but supernovae would succeed. For five objects, gas expulsion is possible neither by winds nor by supernovae.

For each cluster, we performed simulations varying the star formation efficiency by adding a corresponding amount of initial gas to the observed stellar mass. From this we determined the critical star formation efficiency, $\epsilon_{\mathrm{SF}, \mathrm{W}, \mathrm{c}}$, at which the supershell would not escape just yet when driven by the stellar winds. It is also given in Table 1 . We find values as high as 80 per cent.

If the star formation efficiency were indeed to rise until stellar winds are able to clear the remaining gas, this would then occur on a timescale of a few $10^{5} \mathrm{yr}$ (Table 1), which would agree with the observational finding that these clusters are gas-free after a few Myr.

\subsection{Model grid for all cases of energy injection}

We ran a model grid for stellar masses of $10^{5}, 10^{6}$, and $10^{7} \mathrm{M}_{\odot}$ and half-mass radii of 1,3 , and $10 \mathrm{pc}$, varying the star formation efficiency in steps of $\Delta \epsilon_{\mathrm{SF}}=0.1$ for the following energy production models: stellar winds at low metallicity $([\mathrm{Fe} / \mathrm{H}]=-1.5)$, stellar winds at solar metallicity, normal supernovae, hypernovae at $10^{52} \mathrm{erg}$ each, and hypernovae at $10^{53} \mathrm{erg}$ each. For each 
scenario, we determined the critical star formation efficiency for gas expulsion, and, where the grid contained cases of successful gas expulsion, the time for the supershell to reach the half-mass radius. We defined the critical star formation efficiency for gas expulsion $\epsilon_{\mathrm{SF} \text {,crit }}$ as the one where gas expulsion does not succeed just yet. The model with the next higher star formation efficiency (by 0.1 ) would then feature successful gas expulsion. The results are shown in Table 2.

The critical star formation efficiency for this model grid assumes the full range of possible values. It generally increases for increasing mass and decreasing radius. For the low-metallicity winds, even the $10^{5} M_{\odot}$ clusters have critical star formation efficiencies above 30 to 80 per cent. For the $10^{7} M_{\odot}$ clusters, it approaches 100 percent. At solar metallicity, $\epsilon_{\mathrm{SF}, \text { crit }}$ drops to zero for the lightest and most extended cluster, whereas the heavy and concentrated clusters still have values of 90 per cent. The table for normal supernovae is remarkably similar to the table for solar metallicity winds. Each factor of ten in explosion energy changes the values by a similar amount as the change in metallicity in the wind models. Hypernovae with $10^{53} \mathrm{erg}$ each reduce $\epsilon_{\mathrm{SF}, \text { crit }}$ to zero for all $10^{5} M_{\odot}$ clusters and to 80 per cent for the most compact $10^{7} M_{\odot}$ object.

We calculated the time for the shell to reach the half-mass radius as a proxy for the gas expulsion timescale. For models that feature successful gas expulsion, this timescale never exceeds the crossing time by more than a factor of 2.5 and is usually below the crossing time. A much higher star formation efficiency than the critical one, where possible, reduces this timescale further.

In a simple scaling argument, the binding energy of gas in a star cluster is proportional to $\left(1-\epsilon_{S F}\right) M_{\text {tot }}^{2} / r_{\mathrm{h}}$ (compare Sect. 3), where $M_{\text {tot }}$ is the total initial mass. The feedback energy should scale with the stellar mass $M_{*}=\epsilon_{\mathrm{SF}} M_{\mathrm{tot}}$. Gas expulsion should occur when the supplied feedback energy reaches a certain threshold that should scale with the binding energy. This would imply $\left(1-\epsilon_{\mathrm{SF}}\right)\left(M_{*} / \epsilon_{\mathrm{SF}}\right)^{2} / r_{\mathrm{h}} \propto M_{*}$, and hence

$\epsilon_{\mathrm{SF}}=-\frac{a C_{5}}{2}+\sqrt{\frac{a^{2} C_{5}^{2}}{4}+a C_{5}}$,

with the constant of proportionality $a$ and the compactness in$\operatorname{dex} C_{5}=\left(M_{*} / 10^{5} M_{\odot}\right)\left(r_{\mathrm{h}} / \mathrm{pc}\right)^{-1}$. We show the critical star formation efficiency plotted against the compactness index in Fig. 4 together with fits of Eq. (2). Clearly, Eq. (2) describes our models well. We provide the values for the fit parameter $a$ for the respective cases in Table 3.

\section{Discussion}

\subsection{Gas expulsion in young massive clusters}

Five of the YMCs in the sample of Bastian et al. (2014) could have expelled their residual gas neither by stellar winds nor by normal supernovae (and also not by radiation pressure, compare Sect. 3) if the star formation efficiency was 30 per cent, which is the preferred value for self-enrichment scenarios to explain the ratio of first- to second-generation stars. Extrapolating our grid results using Eq. (2) with the parameter $a$ from Table 3, we find that $10^{52}$ erg hypernovae would be needed to expel the gas at this star formation efficiency in one case (T352/W38220) and $10^{53}$ erg hypernovae would be required for the other four clusters.

The agent of the possible gas expulsion can be constrained from the observationally inferred timescales: Stellar explosions
Table 2. Critical star formation efficiency $\epsilon_{\mathrm{SF}, \text { crit }}$ for successful gas removal in a certain scenario.

\begin{tabular}{cccc}
\hline \hline $\log \left(M_{*} / M_{\odot}\right)^{a}$ & $r_{\mathrm{h}}=1 \mathrm{pc}^{b}$ & $r_{\mathrm{h}}=3 \mathrm{pc}^{b}$ & $r_{\mathrm{h}}=10 \mathrm{pc}^{b}$ \\
\hline \multicolumn{4}{l}{ Stellar winds at low metallicity, [Fe/H] $=-1.5$} \\
5 & $0.8(1.45)$ & $0.5(0.73)$ & $0.3(0.37)$ \\
6 & 0.9 & $0.8(1.88)$ & $0.6(0.63)$ \\
7 & 0.9 & 0.9 & 0.9
\end{tabular}

Stellar winds at solar metallicity

$\begin{array}{llll}5 & 0.4(1.90) & 0.3(0.59) & 0(0.63) \\ 6 & 0.9 & 0.8(0.84) & 0.4(0.46) \\ 7 & 0.9 & 0.9 & 0.8(0.63)\end{array}$

Normal supernovae, $E_{0}=10^{51} \mathrm{erg}$

$\begin{array}{llll}5 & 0.5(0.66) & 0.2(0.55) & 0(0.54) \\ 6 & 0.9 & 0.7(0.52) & 0.4(0.37) \\ 7 & 0.9 & 0.9 & 0.8(0.42)\end{array}$

Hypernovae, $E_{0}=10^{52} \mathrm{erg}$

$\begin{array}{llll}5 & 0.2(0.64) & 0(0.72) & 0(0.25) \\ 6 & 0.7(0.56) & 0.3(0.61) & 0.1(0.43) \\ 7 & 0.9 & 0.8(0.52) & 0.4(0.55)\end{array}$

Hypernovae, $E_{0}=10^{53} \mathrm{erg}$

\begin{tabular}{llll}
5 & $0(0.80)$ & $0(0.31)$ & $0(0.11)$ \\
6 & $0.3(0.64)$ & $0.1(0.56)$ & $0(0.36)$ \\
7 & $0.8(0.67)$ & $0.5(0.55)$ & $0.1(0.65)$ \\
\hline
\end{tabular}

Notes. Given is $\epsilon_{\mathrm{SF}}$ of the last model that did not result in successful gas expulsion, where $\epsilon_{\mathrm{SF}}$ was varied in steps of 0.1 , or 0 if all investigated models lead to gas expulsion. If the number is lower than 0.9 , we give the gas removal timescale at a star formation efficiency higher by $\Delta \epsilon_{\mathrm{SF}, \text { crit }}=0.1$ in units of the crossing time in brackets. ${ }^{(a)}$ Base 10 logarithm of the initial stellar mass of the cluster in solar masses. ${ }^{(b)} r_{\mathrm{h}}$ : half-mass radius .

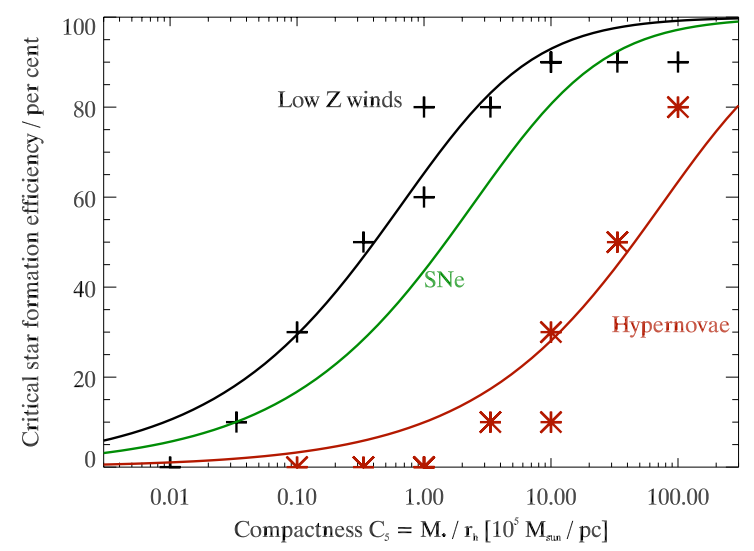

Fig. 4. Critical star formation efficiency for gas expulsion $\epsilon_{\mathrm{SF}, \text { crit }}$ by lowmetallicity stellar winds (black plus-signs) and $10^{53} \mathrm{erg}$ hypernovae (red stars) versus compactness index $C_{5}$. The black and red solid lines are fits made using Eq. (2). The fit to the models with normal supernovae is shown in green for comparison.

appear only after the most massive stars have completed their main-sequence and Wolf-Rayet phases, that is 3-4 Myr after formation. The tightest constraints on timescales probably come 
Table 3. Fit parameter $a$ of Eq. (2) for the different cases for the energy production from Table 2 .

\begin{tabular}{ll}
\hline \hline Case & \multicolumn{1}{c}{$a$} \\
\hline Stellar winds at low metallicity, $[\mathrm{Fe} / \mathrm{H}]=-1.5$ & 1.23 \\
Stellar winds at solar metallicity & 0.368 \\
Normal supernovae, $E_{0}=10^{51} \mathrm{erg}$ & 0.337 \\
Hypernovae, $E_{0}=10^{52} \mathrm{erg}$ & 0.0554 \\
Hypernovae, $E_{0}=10^{53} \mathrm{erg}$ & 0.0110 \\
\hline
\end{tabular}

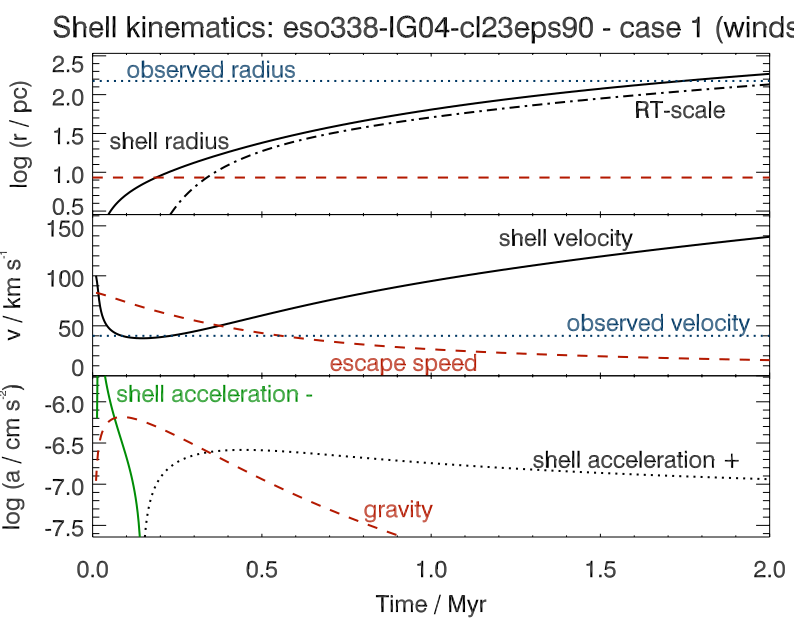

Fig. 5. Shell kinematics for cluster 23 in ESO 338-IG04 for the case where the energy is produced by stellar winds and a star formation efficiency of 90 per cent. The meaning of the lines is the same as in Fig. 3, except for the blue dotted lines, which give the observed radius (upper panel) and velocity (middle panel) of the supershell around this object. The low observed velocity compared to our model would mean that most of the observed shell mass would be contributed by ISM around the cluster rather than gas that was originally deep inside its potential well.

from cluster 23 in ESO 338-IG04 and cluster A in NGC 1569. The former has an age of $6_{-2}^{+4} \mathrm{Myr}$. The surrounding superbubble expands at $\approx 40 \mathrm{~km} \mathrm{~s}^{-1}$ and has a radius of $150 \pm 30 \mathrm{pc}$ (Bastian et al. 2014). The inferred dynamical age of 3-4.5 Myr implies that any residual gas was expelled from the cluster $\approx 0-5.5 \mathrm{Myr}$ after formation of the cluster. Cluster A in NGC 1569 shares a superbubble with a somewhat older neighbouring cluster, thus it could have lost its gas at any point during its lifetime of $6 \pm$ 1 Myr. For both objects, it would therefore be still just possible to lose the gas at the beginning of the supernova phase if the star formation efficiency was high or if the average energy per explosion was higher than $10^{51} \mathrm{erg} \mathrm{s}^{-1}$, although stellar winds appear more likely from the timescale constraints.

One way to remove residual gas is indeed to assume a higher star formation efficiency. In Fig. 5 we show the shell kinematics for the successful gas expulsion case by stellar winds if the star formation efficiency was as high as 90 per cent. The shell would need 1.8 Myr to reach the observed radius, and the velocity would then be $130 \mathrm{~km} \mathrm{~s}^{-1}$, about three times higher than observed. However, the model assumes essentially zero density outside the cluster (Plummer model). The low observed velocity would then imply, as expected, that the shell gas is mainly material that never fell deeply into the star cluster potential. Thus, observations of the shell mass may only place lower limits on the star formation efficiency.

\subsection{Model grid: importance of compactness and star formation efficiency}

The model grids demonstrate that the compactness index, $C_{5}$, is the parameter that governs gas expulsion. The numerical results also show some real scatter, however, which is most likely due to the non-linearity of the underlying physics. While the models shown in Fig. 4 generally follow Eq. (2) well, any two models with the same $C_{5}$ may still differ by 20 percentiles in critical star formation rate.

The critical star formation efficiency we derive could be interpreted in the context of self-regulated star-cluster formation: once enough massive stars are formed, the feedback energy would be sufficient to dispel the remaining gas and terminate star formation. Such a process has been discussed by Zinnecker \& Yorke (2007) for massive star formation in general. Smooth particle hydrodynamics simulations of the formation of star clusters with feedback find a more complex behaviour, where gas clearance is sometimes dominated by accretion of the gas on to stars, but sometimes significant amounts of gas are driven away, depending on the implementation of feedback (Dale 2015).

Supernovae are probably not very important for gas expulsion at solar metallicities or above: the injected power is comparable to that of the stellar winds. The latter act, however, before the supernovae and are therefore more probable to be the actual agents of gas removal, if it is removed.

At low metallicity, supernovae could be important drivers of natal cloud destruction, because, for a given local star formation efficiency, the supernovae inject more power.

If star clusters hosting multiple hypernovae would exist, they would of course be the dominant agents of gas removal. They would be able to cause gas expulsion (local star formation efficiency $<50$ percent) up to a compactness index of $C_{5} \approx 30$. This corresponds to, for example, a $10^{7} M_{\odot}$ star cluster with a half-mass radius of $3 \mathrm{pc}$.

\subsection{Gas expulsion and multiple populations}

\subsubsection{Constraints from $\mathrm{N}$-body simulations}

Khalaj \& Baumgardt (2015) show $N$-body simulations of star clusters that contain chemically normal (first generation) and peculiar (second generation) stars. With a simple model of gas expulsion they find that star formation efficiencies $\lesssim 50$ per cent are needed in order to produce star clusters with final ratios of first to second generation stars close to the ones observed in presentday globular clusters in the Milky Way. Our model grid results imply that this would only be possible if the compactness indices $C_{5}$ would not exceed unity (or up to 30 , if series of strong hypernovae would occur in such clusters).

We collected parameters for clusters that have been searched for multiple populations from the literature (Table B.1), and calculated their $C_{5}$ value. We included both, clusters with detailed abundance analysis, as well as ones that show multiple populations in the colour-magnitude diagrams.

Current $C_{5}$-values extend up to a few for objects that do show multiple populations (they are all globular clusters). This would already put them into a regime where gas expulsion by stellar winds and supernovae would not have been possible. However, their masses must have been even higher, and the radii smaller, if gas expulsion would indeed have been at work in these objects: Bastian \& Lardo (2015) compare the first to second generation ratios predicted by Khalaj \& Baumgardt (2015) to observations and find that all clusters that show the 
M. G. H. Krause et al.: Gas expulsion in massive star clusters?
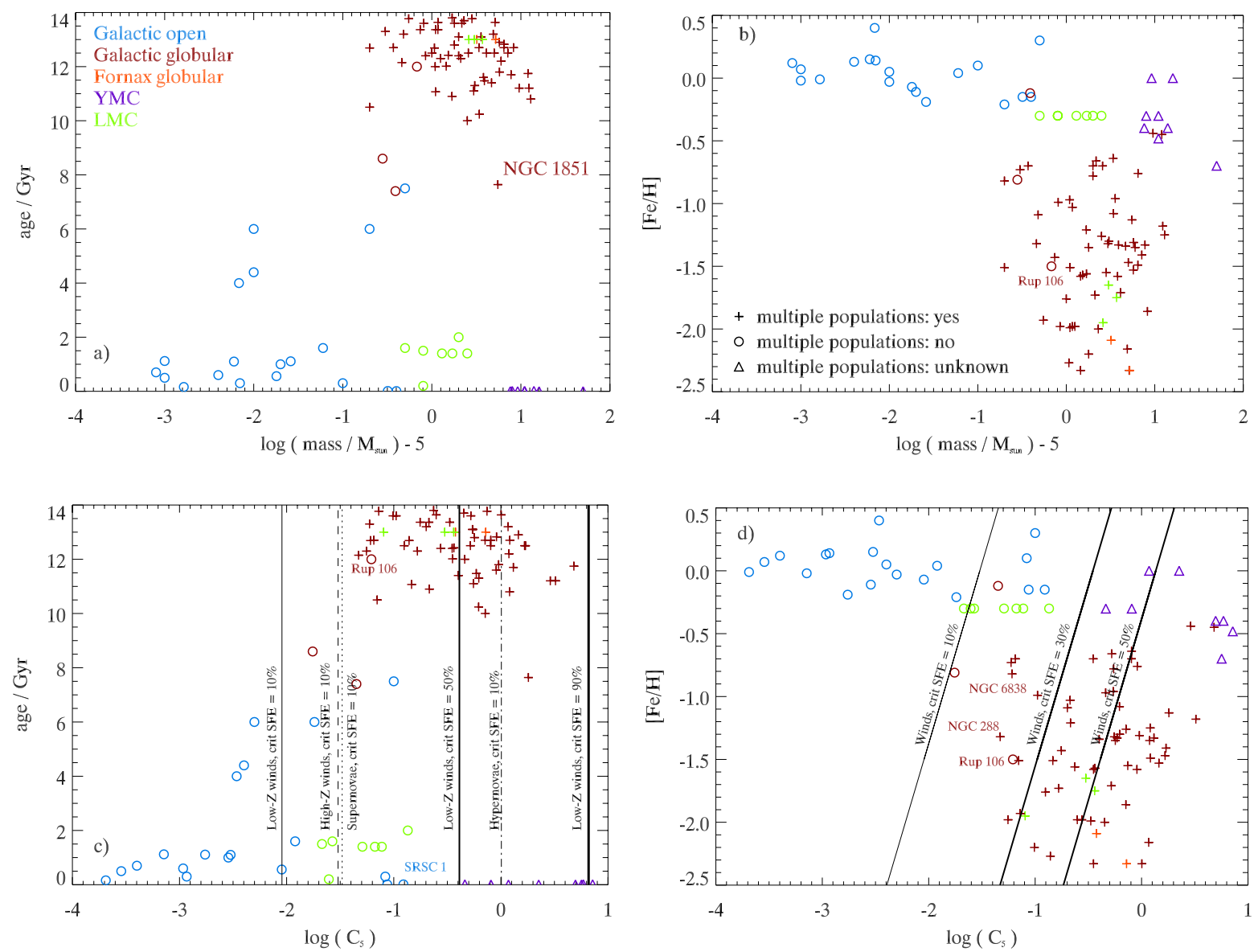

Fig. 6. Star clusters from Tables 1 and B.1 in different parameter spaces. Different symbols are used according to the presence of multiple populations, as indicated either from photometry or spectroscopic abundance analysis: we indicate star clusters that have been searched for multiple populations but with negative result (open circles), with positive result (plus signs), and clusters where the presence of multiple populations is not known (triangles). The colours code different samples: GCs: dark red, Fornax globular clusters: light red, YMCs: purple, LMC clusters: green, Galactic open clusters: blue. Some star clusters discussed in the text are highlighted. a) Mass-age diagram. b) Mass-metallicity diagram. c) Compactness-age diagram. Black vertical lines indicate the critical star formation efficiency required to expel the remaining gas as calculated in the present work. Solid lines are for the low-metallicity stellar winds models (thin: 10 per cent, medium: 50 per cent, thick: 90 per cent). Other lines are for 10 per cent critical star formation efficiency; the dashed line for solar metallicity winds, the dotted line for normal supernovae, and the dot-dashed line for hypernovae of $10^{53} \mathrm{erg}$, each. d) Compactness-metallicity diagram. Solid lines separate regions where gas expulsion is possible with the indicated star formation efficiency (left) from regions where this is not possible (right), according to the present work, for the case of metallicity dependent stellar winds.

$\mathrm{Na}-\mathrm{O}$ anti-correlation should have lost a very similar fraction of stars, between 95 and 98 per cent of the initial number (compare also Prantzos \& Charbonnel 2006; Carretta et al. 2010; D'Ercole et al. 2010). Expansion factors are up to a few (Khalaj $\&$ Baumgardt 2015). The current $C_{5}$-values, 0.05-3.2, would hence transform to the range of about 1 to 100 for the initial compactness indices. Hence, even a series of very energetic events, like hypernovae, could not have been the agent of gas expulsion in the most compact objects. This is in the context of early gas expulsion models, motivated by the compact, young, gasfree clusters discussed earlier. Energetic events late in the evolution of a star cluster have been discussed elsewhere (Paper I), and would also be a possible agent for gas expulsion in young GCs, if the results from YMCs (gas-free after a few Myr) would for some reason not apply to clusters that show light-element anti-correlations.

Summarising, these results suggest that gas expulsion is not a viable solution to the mass budget problem in the context of multiple population star clusters. A similar conclusion was reach with different arguments by Khalaj \& Baumgardt (2015) and Bastian \& Lardo (2015).

\subsubsection{Parameter space for abundance anomalies}

We compare the parameter space occupied by star clusters with and without evidence for multiple populations to the ones where gas expulsion may occur in more detail in Fig. 6. Apart from a possible early gas expulsion event, star clusters lose mass over time for instance as a result of low-mass star depletion (Kruijssen \& Mieske 2009), encounters with the environment (Kruijssen et al. 2012), or disc passages (Webb et al. 2014), which may also lead to expansion through tidal heating. Hence, we generally expect that $C_{5}$ will decrease with time, easily by a factor of a few and very likely in different ways for different objects (e.g., Rossi \& Hurley 2015b). For example, Rossi \& Hurley (2015a) reconstructed the mass-loss history of the two Milky Way GCs HP 1 and NGC 6553. According to these calculations, the former GC experienced a secular mass loss of 79 per cent of its initial mass, the latter one even 93 per cent. Recent observational studies typically have abundance uncertainties less than 0.1 dex (e.g., Mucciarelli et al. 2011; Villanova et al. 2013). Mass, age and radius accuracies are of the order of 10 per cent (Bastian et al. 2014). Absolute ages for GCs have 
a systematic uncertainty (Carretta et al. 2010). Therefore, systematic effects should dominate, but measurement uncertainties might also be important in some cases, especially for the older studies.

The mass-age diagram is shown in Fig. 6a. With the addition of the relatively recent data from the Large Magellanic Cloud (LMC, green), the overlap in mass between clusters that do or do not have multiple populations has become substantial. It is now more than one dex, suggesting that at least a second parameter is required. The latter could be age, with multiple populations being restricted to old star clusters. Clear outliers would be the single population cluster Rup 106 with an age of $12 \mathrm{Gyr}$, and the multiple population cluster NGC 1851 at 8 Gyr.

The second parameter could also be metallicity (Fig. 6b). In this case, star clusters with $[\mathrm{Fe} / \mathrm{H}]$ below -0.5 would have multiple populations. Outliers in this case would be the single population clusters Pal $12([\mathrm{Fe} / \mathrm{H}]=-0.8)$ and Rup 106 $([\mathrm{Fe} / \mathrm{H}]=-1.5)$. Especially the latter is located far inside the region of parameter space occupied by multiple-population clusters.

When considering the compactness index $C_{5}$ instead of the mass as fundamental parameter for the expression of multiple populations (Fig. 6c), star clusters with and without multiple populations are also not cleanly separated. The overlap is, however, reduced: the most compact single population cluster in our sample is the Scutum red supergiant cluster with $\log \left(C_{5}\right)=-0.92$. The least compact multiple population cluster is NGC 288 with $\log \left(C_{5}\right)=-1.30$. Thus, the overlap region is much smaller compared to the case where mass is taken as the primary parameter. Again, age may be taken as additional parameter to separate single and multiple population clusters with the same caveat as above.

Star clusters with and without multiple populations separate more clearly in the compactness-metallicity diagram (Fig. 6d) compared to the mass-metallicity diagram. In particular, Rup 106 has now moved towards the left border of the region occupied by multiple population clusters.

Rup 106 is a particularly well studied object (Dotter et al. 2011; Villanova et al. 2013): Its age is accurately known from Hubble Space Telescope $\mathrm{ACS}^{1}$ photometry. Abundances for nine stars have been measured with the high resolution spectrograph $\mathrm{UVES}^{2}$ on the Very Large Telescope. The probability to have missed a second population with similar properties than in other GCs is below $10^{-4}$. Hence, it is not an outlier with large observational uncertainties.

Critical star formation efficiencies for gas expulsion are indicated in Figs. 6c and d. Considering current parameters, single population clusters prefer regions of parameter space where gas expulsion would be easily possible from our analysis. Multiple population star clusters extend into regions of parameter space, where stellar wind and supernova feedback could only remove the residual gas when the star formation efficiency was above 50 per cent - too much for gas expulsion with significant loss of stars. This conclusion is reinforced when considering that evolutionary effects tend to increase the radii and decrease the masses of star clusters, i.e. they have typically been more compact in the past.

The critical star formation efficiencies for metallicity dependent stellar winds is indicated by the oblique lines in Fig. $6 \mathrm{~d}$. These lines again do not cleanly separate single from multiple population clusters. They do, however, further reduce the

\footnotetext{
1 Advanced Camera for Surveys.

2 Ultraviolet and Visual Echelle Spectrograph.
}

overlap in $C_{5}$ from 0.38 dex to about 0.3 dex. Hence, a scenario, where multiple populations are suppressed whenever the compactness is low enough so that stellar winds can expel the gas, best explains the data.

It is instructive to compare Rup 106 to NGC 6535. Rup 106 has been mentioned above as a well-studied single population GC. It is marked in Fig. 6d, and sits marginally, but clearly, in the parameter space occupied by multiple-population clusters. NGC 6535 is a multiple-population cluster with essentially the same $C_{5}$ and metallicity than Rup 106 . Consequently, their symbols partially overlap in Fig. 6d. NGC 6535 has a Galactocentric distance (Harris 1996) of $3.9 \mathrm{kpc}$, hence can be expected to have experience strong mass loss due to tidal interactions with disc and bar (Rossi \& Hurley 2015a,b). Its initial $C_{5}$ was therefore likely much higher, i.e. it would have been found more to the right, in the cloud of the other multiple-population clusters. Rup 106 has a Galactocentric distance of $18.5 \mathrm{kpc}$. Therefore, secular mass loss was probably lower, and its initial location in Fig. 6d was likely not very different from the current one. The situation is similar for the multiple-population GCs with similar $C_{5}$ and higher metallicity than these two objects (pluses above them in Fig. 6d): they are all at rather low Galactocentric distances (3.3 to 6.7) kpc. An exception is NGC 288 at $12 \mathrm{kpc}$, also accurately measured.

These considerations are consistent with the aforementioned expectation that systematic errors due to secular evolution dominate the uncertainties. The small overlap of 0.3 dex found above for a separation due to gas expulsion by stellar wind feedback agrees quantitatively with the expected magnitude of the systematic uncertainties. Hence, the hypothesis describes the data well within the general limitations mentioned above.

The YMCs from the sample of Bastian et al. (2014) would form a powerful test for a possible connection between gas expulsion and the expression of multiple populations. Some of these objects have even higher $C_{5}$ than the most compact GCs. They also join the GCs smoothly in the $[\mathrm{Fe} / \mathrm{H}]$ over $C_{5}$ plot, so that one would expect them to show anti-correlations, based on these parameters. If they were not to show the anti-correlation, it would be clear that the physics of the clearing of the residual gas is not related to it or that YMCs cannot be considered as the counterpart of young GCs.

\subsection{Accuracy of the method}

We presented a number of thin-shell models for gas expulsion, where gas expulsion was deemed successful if the solution passed a Rayleigh-Taylor criterion.

These models are simplifications of the 3D hydrodynamic problem that allowed calculating a large number of models. A 3D initial condition will entail some degree of clumping of the gas. During the early embedded phase, this gas is likely to continue gravitational contraction, whereas the diffuse component is shifted into shells, as is also seen in 3D hydrodynamics simulations of low-mass clusters (e.g., Dale \& Bonnell 2012). The clumped gas that is about to form stars contributes to the gravitational potential without producing feedback energy, which makes it more difficult for the remaining gas to escape. On the other hand, there is effectively less gas to expel, which makes it easier for the given amount of feedback energy to accelerate the reduced gas mass. The overall effect might thus not be very strong. However, 3D simulations are now necessary to assess this quantitatively. If the gas were to remain in the clusters for long enough to be affected by supernovae (a few Myr), the denser clumps would most likely already have formed stars or 
protostellar cores, and the remaining gas is expected to be reasonably smooth, such that spherical symmetry would again most likely be a reasonable assumption.

The largest uncertainty probably concerns the initial conditions. The assumption that the gas is distributed radially like the stars is not expected to hold. For hydrostatic equilibrium it would require pressure support. Thermal pressure would be unlikely because of the strong cooling expected at the implied gas densities. Magnetic fields would probably lead to compression into filaments and then to star formation. Radiation pressure has been shown in Sect. 3 to be insufficient. Turbulence could support the gas for about one crossing time (e.g., Elmegreen \& Scalo 2004). Hence, we expect the gas generally to be in an inflow or outflow state.

During the formation of the stars and shortly afterwards, we expect the gas-to-star ratio in the centre of the cluster to be somewhat lower than what we assumed because of the gas depletion by star formation (e.g., Kruijssen et al. 2012). If the gas is not expelled early, then we might expect the gas-to-star ratio in the centre to be higher than what we assumed later on as a result of accumulation in the centre. Generally, the gas is likely to have an inward velocity because it is, of course, accumulating to form a star cluster. Except perhaps for the earliest phases, these effects would make gas expulsion more difficult than is implied by the present models.

Some recent 3D hydrodynamics simulations have addressed already a few issues present in our simple models: Distributed energy/momentum sources lead to enhanced dissipation (Krause et al. 2013b; Bourne \& Power 2016). This is again a factor that makes it easier for gas expulsion to occur in our simple models, where energy input effectively happens at the cluster centre.

Calura et al. (2015) simulate an embedded star cluster with compactness index $C_{5}=0.85$ and a metallicity of $[\mathrm{Fe} / \mathrm{H}]=$ -1.3 . For these parameters, our simple model predicts a minimum local star formation efficiency for gas expulsion on the crossing timescale of 0.61 in the wind phase, and, respectively 0.41 in the supernova phase. The authors use a star formation efficiency of 0.4. They do not find gas expulsion on the crossing timescale, but a slow mass loss with a characteristic timescale of $10 \mathrm{Myr}$, entirely consistent with the prediction of our simple model.

We are therefore confident that our models are able to reasonably rule out gas expulsion for certain star cluster parameters. However, star clusters for which our model predicts gas expulsion might still be able to retain the gas, for example because the gas distribution is more concentrated than assumed in the model.

\section{Conclusions}

The gas expulsion paradigm for massive star clusters is currently debated in the literature. We investigated the conditions for gas expulsion in massive star clusters in the context of different observational constraints by means of a large number of self-gravitating thin-shell models and an analytical estimate for the stability of the shell.

For several extragalactic, young massive clusters, we found that gas expulsion would not have been possible for standard assumptions, including an IMF according to Kroupa et al. (2013), a star formation efficiency of 30 per cent, and the assumption that 20 per cent of the energy produced by stellar feedback couples to the gas. The result holds for both, stellar winds and supernovae as energy sources. Radiation pressure is not important in this context. Yet, these clusters are gas-free at an age of around 10 Myr.
We then showed that the gas could be cleared if the star formation efficiency would be increased. For some clusters the star formation efficiencies would have to be higher than 80 per cent to remove the rest of the gas. This, however, would be too high for any significant change of the gravitational potential. Hence, the stellar mass could not be significantly affected by this kind of gas loss. Thus, for canonical energy production and IMF, the standard paradigm of residual gas expulsion (implying a substantial loss of stars) cannot work in these clusters.

With a grid of star clusters with masses between $10^{5} M_{\odot}$ and $10^{7} M_{\odot}$, and half-mass radii between 1 and $10 \mathrm{pc}$, we showed that gas expulsion, if successful, indeed happens on the crossing timescale. This confirms that the supershell ansatz is indeed a useful realisation for gas expulsion on the crossing timescale.

The critical star formation efficiency required to remove the remaining gas is a function of the compactness index $C_{5} \equiv$ $\left(M_{*} / 10^{5} M_{\odot}\right) /\left(r_{\mathrm{h}} / \mathrm{pc}\right)$. More compact clusters (higher $\left.C_{5}\right)$ would transform relatively more gas into stars. Any gas loss in massive, compact star clusters would therefore be moderate, and could not lead to strong losses of stars, in good agreement with a recent census of GCs and stars in Fornax (Larsen et al. 2014b). This is, however, a problem for models that try to explain the observed ratio of first to second generation stars in globular clusters by a gas-expulsion induced loss of first generation stars.

We showed by comparison to observations that compactness is a better predictor for the occurrence of multiple populations than mass. This suggests that the absence of gas expulsion might play a role for the expression of the $\mathrm{Na}-\mathrm{O}$ anti-correlations and multiple populations in globular clusters. The best separation between clusters that do and ones that do not have multiple populations is achieved by a model for gas expulsion based on metallicity dependent stellar winds.

Gas expulsion scenarios that have been developed to reproduce the light element anti-correlations in globular clusters would require initial compactness indices of $1 \lesssim C_{5} \lesssim 100$. In Paper I, energetic events at late times were proposed as a solution to this problem. Observations of YMCs suggest, however, that the gas is lost early in the evolution of also massive star clusters. Here, we showed that early gas expulsion, cannot work for most of this parameter range. On the basis of this argument, gas expulsion cannot be the solution of the mass budget problem for the expression of multiple populations in globular clusters.

Acknowledgements. We thank Cyril Georgy for providing data for rotating star models, and Georges Meynet and Andrii Neronov for helpful discussions. This work was supported by funding from Deutsche Forschungsgemeinschaft under DFG project number PR 569/10-1 in the context of the Priority Program 1573 "Physics of the Interstellar Medium" and the International Space Science Institute (International Team 271 "Massive Star Cluster across the Hubble Time"). Additional support came from funds from the Munich Cluster of Excellence "Origin and Structure of the Universe". (http://www . universe-cluster.de). C.C. acknowledges financial support from the Swiss National Science Foundation (FNS) for the project 200020-140346 "New perspectives on the chemical and dynamical evolution of globular clusters in light of new generation stellar models" (PI C.C.). We thank the International Space Science Institute (ISSI, Bern, CH) for welcoming the activities of ISSI Team 271 "Massive Star Clusters across the Hubble Time" (2013-2015; team leader C.C.). We thank the anonymous referees for very useful comments that helped to improve the presentation of these results.

\section{References}

Acreman, D. M., Dobbs, C. L., Brunt, C. M., \& Douglas, K. A. 2012, MNRAS, 422, 241

Banerjee, S., \& Kroupa, P. 2015, Arxiv e-prints [arXiv: 1512.03074]

Banerjee, S., \& Kroupa, P. 2016, A\&A, accepted

Bastian, N., \& Silva-Villa, E. 2013, MNRAS, 431, L122 
Bastian, N., \& Goodwin, S. P. 2006, MNRAS, 369, L9 Bastian, N., \& Lardo, C. 2015, MNRAS, 453, 357 Bastian, N., \& Niederhofer, F. 2015, MNRAS, 448, 1863 Bastian, N., Gieles, M., Goodwin, S. P., et al. 2008, MNRAS, 389, 223 Bastian, N., Lamers, H. J. G. L. M., de Mink, S. E., et al. 2013, MNRAS, 436,

Bastian, N., Hollyhead, K., \& Cabrera-Ziri, I. 2014, MNRAS, 445, 378 Baumgardt, H., \& Kroupa, P. 2007, MNRAS, 380, 1589

Baumgardt, H., Kroupa, P., \& Parmentier, G. 2008, MNRAS, 384, 1231

Becker, W., \& Fenkart, R. 1971, A\&AS, 4, 241

Blandford, R. D., \& Znajek, R. L. 1977, MNRAS, 179, 433

Bourne, M. A., \& Power, C. 2016, MNRAS, 456, L20

Boyles, J., Lorimer, D. R., Turk, P. J., et al. 2011, ApJ, 742, 51

Bragaglia, A., \& Tosi, M. 2006, AJ, 131, 1544

Bragaglia, A., Gratton, R. G., Carretta, E., et al. 2012, A\&A, 548, A122

Bragaglia, A., Sneden, C., Carretta, E., et al. 2014, ApJ, 796, 68

Bragaglia, A., Carretta, E., Sollima, A., et al. 2015, A\&A, 583, A69

Bressert, E., Bastian, N., Gutermuth, R., et al. 2010, MNRAS, 409, L54

Brodie, J. P., \& Strader, J. 2006, ARA\&A, 44, 193

Brown, J. H., Burkert, A., \& Truran, J. W. 1995, ApJ, 440, 666

Cabrera-Ziri, I., Bastian, N., Longmore, S. N., et al. 2015, MNRAS, 448, 2224

Caloi, V., \& D'Antona, F. 2011, MNRAS, 417, 228

Calura, F., Few, C. G., Romano, D., \& D'Ercole, A. 2015, ApJ, 814, L14

Cantat-Gaudin, T., Vallenari, A., Zaggia, S., et al. 2014, A\&A, 569, A17

Carretta, E., Bragaglia, A., Gratton, R. G., et al. 2010, A\&A, 516, A55

Casewell, S. L., Littlefair, S. P., Burleigh, M. R., \& Roy, M. 2014, MNRAS, 441, 2644

Cassisi, S., \& Salaris, M. 2014, A\&A, 563, A10

Charbonnel, C. 2010, in IAU Symp. 266, eds. R. de Grijs, \& J. R. D. Lépine, 131

Charbonnel, C., Chantereau, W., Krause, M., Primas, F., \& Wang, Y. 2014, A\&A, 569, L6

Christodoulou, D. 1970, Phys. Rev. Lett., 25, 1596

Clarkson, W. I., Ghez, A. M., Morris, M. R., et al. 2012, ApJ, 751, 132

Conroy, C. 2012, ApJ, 758, 21

Cottaar, M., \& Hénault-Brunet, V. 2014, A\&A, 562, A20

Cottaar, M., Meyer, M. R., Andersen, M., \& Espinoza, P. 2012, A\&A, 539, A5

Dale, J. E. 2015, New Astron. Rev., 68, 1

Dale, J. E., \& Bonnell, I. A. 2012, MNRAS, 422, 1352

Dale, J. E., Ngoumou, J., Ercolano, B., \& Bonnell, I. A. 2014, MNRAS, 442, 694

Dale, J. E., Ercolano, B., \& Bonnell, I. A. 2015, MNRAS, 451, 987

Davies, B., Origlia, L., Kudritzki, R.-P., et al. 2009, ApJ, 696, 2014

de Mink, S. E., Pols, O. R., Langer, N., \& Izzard, R. G. 2009, A\&A, 507, L1

Decressin, T., Meynet, G., Charbonnel, C., Prantzos, N., \& Ekström, S. 2007, A\&A, 464, 1029

Decressin, T., Baumgardt, H., Charbonnel, C., \& Kroupa, P. 2010, A\&A, 516, A73

Denissenkov, P. A., \& Hartwick, F. D. A. 2014, MNRAS, 437, L21

D’Ercole, A., Vesperini, E., D’Antona, F., McMillan, S. L. W., \& Recchi, S. 2008, MNRAS, 391, 825

D’Ercole, A., D’Antona, F., Ventura, P., Vesperini, E., \& McMillan, S. L. W. 2010, MNRAS, 407, 854

D'Ercole, A., D'Antona, F., \& Vesperini, E. 2011, MNRAS, 415, 1304

Donati, P., Beccari, G., Bragaglia, A., Cignoni, M., \& Tosi, M. 2014, MNRAS, 437, 1241

Dotter, A., Sarajedini, A., \& Anderson, J. 2011, ApJ, 738, 74

Ekström, S., Georgy, C., Eggenberger, P., et al. 2012, A\&A, 537, A146

Elmegreen, B. G., \& Scalo, J. 2004, Annu. Rev. Astron. Astrophys., 42, 211

Fenner, Y., Campbell, S., Karakas, A. I., Lattanzio, J. C., \& Gibson, B. K. 2004, MNRAS, 353, 789

Ferraro, F. R., Mucciarelli, A., Carretta, E., \& Origlia, L. 2006, ApJ, 645, L33

Fierlinger, K. M., Burkert, A., Diehl, R., et al. 2012, in Advances in computational astrophysics: methods, tools, and outcome, eds. R. Capuzzo Dolcetta, M. Limongei, A. Tomambe, \& G. Giobbi, ASP Conf. Ser., 453

Forbes, D. A., \& Bridges, T. 2010, MNRAS, 404, 1203

Francic, S. P. 1989, AJ, 98, 888

Freyer, T., Hensler, G., \& Yorke, H. W. 2003, ApJ, 594, 888

Galván-Madrid, R., Liu, H. B., Zhang, Z.-Y., et al. 2013, ApJ, 779, 121

Gebran, M., Monier, R., \& Richard, O. 2008, A\&A, 479, 189

Georgy, C., Ekström, S., Eggenberger, P., et al. 2013, A\&A, 558, A103

Gieles, M., \& Bastian, N. 2008, A\&A, 482, 165

Gieles, M., Sana, H., \& Portegies Zwart, S. F. 2010, MNRAS, 402, 1750

Goodwin, S. P. 1997a, MNRAS, 284, 785

Goodwin, S. P. 1997b, MNRAS, 286, 669

Goodwin, S. P. \& Bastian, N. 2006, MNRAS, 373, 752

Goudfrooij, P., Girardi, L., Kozhurina-Platais, V., et al. 2014, ApJ, 797, 35

Gozha, M. L., Borkova, T. V., \& Marsakov, V. A. 2012, Astron. Lett., 38, 506

Gratton, R. G., Bonifacio, P., Bragaglia, A., et al. 2001, A\&A, 369, 87
Gratton, R. G., Carretta, E., \& Bragaglia, A. 2012, A\&ARv, 20, 50 Güneş, O., Karataş, Y., \& Bonatto, C. 2012, New Astron., 17, 720 Hardcastle, M. J., \& Krause, M. G. H. 2013, MNRAS, 430, 174 Harris, W. E. 1996, AJ, 112, 1487

Hawking, S. W. 1972, Comm. Math. Phys., 25, 152

Heesen, V., Brinks, E., Krause, M. G. H., et al. 2015, MNRAS, 447, L1

Hénault-Brunet, V., Evans, C. J., Sana, H., et al. 2012, A\&A, 546, A73

Heyl, J. S., Richer, H., Anderson, J., et al. 2012, ApJ, 761, 51

Hollyhead, K., Bastian, N., Adamo, A., et al. 2015, MNRAS, 449, 1106

Hurley, J. R., Pols, O. R., Aarseth, S. J., \& Tout, C. A. 2005, MNRAS, 363, 293

Huxor, A. P., Mackey, A. D., Ferguson, A. M. N., et al. 2014, MNRAS, 442, 2165

Janka, H.-T., \& Mueller, E. 1996, A\&A, 306, 167

Khalaj, P., \& Baumgardt, H. 2015, MNRAS, 452, 924

Kimmig, B., Seth, A., Ivans, I. I., et al. 2015, AJ, 149, 53

Klaassen, P. D., Mottram, J. C., Dale, J. E., \& Juhasz, A. 2014, MNRAS, 441, 656

Koleva, M., Prugniel, P., Ocvirk, P., Le Borgne, D., \& Soubiran, C. 2008, MNRAS, 385, 1998

Krause, M. 2005, A\&A, 436, 845

Krause, M. G. H., \& Diehl, R. 2014, ApJ, 794, L21

Krause, M., Charbonnel, C., Decressin, T., et al. 2012, A\&A, 546, L5

Krause, M., Charbonnel, C., Decressin, T., Meynet, G., \& Prantzos, N. 2013a, A\&A, 552, A121

Krause, M., Fierlinger, K., Diehl, R., et al. 2013b, A\&A, 550, A49

Krause, M., Diehl, R., Böhringer, H., Freyberg, M., \& Lubos, D. 2014, A\&A, 566, A94

Kroupa, P., Weidner, C., Pflamm-Altenburg, J., et al. 2013, in Planets, Stars and Stellar Systems, eds. T. Oswalt, \& G. Gilmore (The Netherlands: Springer), 115

Kruijssen, J. M. D., \& Mieske, S. 2009, A\&A, 500, 785

Kruijssen, J. M. D., Maschberger, T., Moeckel, N., et al. 2012, MNRAS, 419, 841

Krumholz, M. R., \& Thompson, T. A. 2013, MNRAS, 434, 2329

Krumholz, M. R., Bate, M. R., Arce, H. G., et al. 2014, in Protostars and Planets VI, ed. H. Beuther, R. S. Klessen, C. P. Dullemond, \& T. Henning (The University of Arizona press), 243

Kuiper, L., \& Hermsen, W. 2015, MNRAS, 449, 3827

Lada, C. J., \& Lada, E. A. 2003, ARA\&A, 41, 57

Langer, N. 2012, ARA\&A, 50, 107

Lanzoni, B., Mucciarelli, A., Origlia, L., et al. 2013, ApJ, 769, 107

Larsen, S. S., Brodie, J. P., \& Strader, J. 2012, A\&A, 546, A53

Larsen, S. S., Brodie, J. P., Forbes, D. A., \& Strader, J. 2014a, A\&A, 565, A98

Larsen, S. S., Brodie, J. P., Grundahl, F., \& Strader, J. 2014b, ApJ, 797, 15

Lebzelter, T., \& Wood, P. R. 2011, A\&A, 529, A137

Lee, S. H., Kang, Y.-W., \& Ann, H. B. 2013, MNRAS, 432, 1672

Leigh, N. W. C., Giersz, M., Marks, M., et al. 2015, MNRAS, 446, 226

Lodieu, N., Boudreault, S., \& Béjar, V. J. S. 2014, MNRAS, 445, 3908

Longmore, S. N., Kruijssen, J. M. D., Bastian, N., et al. 2014, in Protostars and Planets VI, eds. H. Beuther, R. S. Klessen, C. P. Dullemond, \& T. Henning (The University of Arizona press), 291

Lützgendorf, N., Kissler-Patig, M., Gebhardt, K., et al. 2013, A\&A, 552, A49 Mackey, A. D., \& Gilmore, G. F. 2003, MNRAS, 338, 85

Mackey, A. D., Huxor, A. P., Ferguson, A. M. N., et al. 2010, ApJ, 717, L11

Mackey, A. D., Huxor, A. P., Ferguson, A. M. N., et al. 2013, MNRAS, 429, 281

Mackey, A. D., Lewis, G. F., Collins, M. L. M., et al. 2014, MNRAS, 445, L89

MacLean, B. T., De Silva, G. M., \& Lattanzio, J. 2015, MNRAS, 446, 3556

Maeder, A., \& Meynet, G. 2006, A\&A, 448, L37

Maeder, A., \& Meynet, G. 2012, Rev. Mod. Phys., 84, 25

Mandushev, G., Staneva, A., \& Spasova, N. 1991, A\&A, 252, 94

Marconi, G., Hamilton, D., Tosi, M., \& Bragaglia, A. 1997, MNRAS, 291, 763

Marks, M., \& Kroupa, P. 2010, MNRAS, 406, 2000

Marks, M., Kroupa, P., Dabringhausen, J., \& Pawlowski, M. S. 2012, MNRAS, 422, 2246

Mikolaitis, Š., Tautvaišienè, G., Gratton, R., Bragaglia, A., \& Carretta, E. 2010, MNRAS, 407, 1866

Mikolaitis, Š., Tautvaišienè, G., Gratton, R., Bragaglia, A., \& Carretta, E. 2011 MNRAS, 413, 2199

Mikolaitis, Š., Tautvaišienè, G., Gratton, R., Bragaglia, A., \& Carretta, E. 2012, A\&A, 541, A137

Mazzali, P. A., McFadyen, A. I., Woosley, S. E., Pian, E., \& Tanaka, M. 2014, MNRAS, 443, 67

Milone, A. P., Bedin, L. R., Piotto, G., \& Anderson, J. 2009, A\&A, 497, 755

Miocchi, P. 2007, MNRAS, 381, 103

Montes, M., Acosta-Pulido, J. A., Prieto, M. A., \& Fernández-Ontiveros, J. A 2014, MNRAS, 442, 1350

Mucciarelli, A., Ferraro, F. R., Origlia, L., \& Fusi Pecci, F. 2007, AJ, 133, 2053 
M. G. H. Krause et al.: Gas expulsion in massive star clusters?

Mucciarelli, A., Carretta, E., Origlia, L., \& Ferraro, F. R. 2008, AJ, 136, 375

Mucciarelli, A., Origlia, L., Ferraro, F. R., \& Pancino, E. 2009, ApJ, 695, L134

Mucciarelli, A., Origlia, L., \& Ferraro, F. R. 2010, ApJ, 717, 277

Mucciarelli, A., Cristallo, S., Brocato, E., et al. 2011, MNRAS, 413, 837

Mucciarelli, A., Dalessandro, E., Ferraro, F. R., Origlia, L., \& Lanzoni, B. 2014, ApJ, 793, L6

Mucciarelli, A., Lapenna, E., Massari, D., Ferraro, F. R., \& Lanzoni, B. 2015, ApJ, 801, 69

Murray, N., Quataert, E., \& Thompson, T. A. 2010, ApJ, 709, 191

Niederhofer, F., Hilker, M., Bastian, N., \& Silva-Villa, E. 2015, A\&A, 575, A62

Oh, S., Kroupa, P., \& Pflamm-Altenburg, J. 2015, ApJ, 805, 92

Palouš, J., Wünsch, R., Martínez-González, S., et al. 2013, ApJ, 772, 128

Pancino, E., Carrera, R., Rossetti, E., \& Gallart, C. 2010, A\&A, 511, A56

Parmentier, G., \& Gilmore, G. 2007, MNRAS, 377, 352

Penrose, R. 1972, Nature, 236, 377

Peters, T., Schleicher, D. R. G., Klessen, R. S., et al. 2012, ApJ, 760, L28

Pfalzner, S., \& Kaczmarek, T. 2013, A\&A, 559, A38

Pfalzner, S., Parmentier, G., Steinhausen, M., Vincke, K., \& Menten, K. 2014, ApJ, 794, 147

Piatek, S., Pryor, C., McClure, R. D., Fletcher, J. M., \& Hesser, J. E. 1994, AJ, 107,1397

Piotto, G., Milone, A. P., Bedin, L. R., et al. 2015, AJ, 149, 91

Platais, I., Cudworth, K. M., Kozhurina-Platais, V., et al. 2011, ApJ, 733, L1

Portegies Zwart, S. F., McMillan, S. L. W., Hut, P., \& Makino, J. 2001, MNRAS, 321,199

Portegies Zwart, S. F., McMillan, S. L. W., \& Gieles, M. 2010, ARA\&A, 48, 431

Poujade, O., \& Peybernes, M. 2010, Phys. Rev. E, 81, 016316

Prantzos, N., \& Charbonnel, C. 2006, A\&A, 458, 135

Prantzos, N., Charbonnel, C., \& Iliadis, C. 2007, A\&A, 470, 179

Preibisch, T., Roccatagliata, V., Gaczkowski, B., \& Ratzka, T. 2012, A\&A, 541, A132

Reddy, A. B. S., Giridhar, S., \& Lambert, D. L. 2012, MNRAS, 419, 1350

Rochau, B., Brandner, W., Stolte, A., et al. 2010, ApJ, 716, L90
Rossi, L. J., \& Hurley, J. R. 2015a, MNRAS, 454, 1453

Rossi, L. J., \& Hurley, J. R. 2015b, MNRAS, 446, 3389

Salaris, M., \& Weiss, A. 2002, A\&A, 388, 492

Santrich, O. J. K., Pereira, C. B., \& Drake, N. A. 2013, A\&A, 554, A2

Schaerer, D., \& Charbonnel, C. 2011, MNRAS, 413, 2297

Schartmann, M., Burkert, A., Krause, M., et al. 2010, MNRAS, 403, 1801

Shull, J. M., \& Saken, J. M. 1995, ApJ, 444, 663

Sills, A., \& Glebbeek, E. 2010, MNRAS, 407, 277

Smiljanic, R., Gauderon, R., North, P., et al. 2009, A\&A, 502, 267

Strickland, D. K., \& Heckman, T. M. 2009, ApJ, 697, 2030

Tadross, A. L. 2001, New Astron., 6, 293

Tenorio-Tagle, G., Muñoz-Tuñón, C., Silich, S., \& Cassisi, S. 2015, ApJ, 814, L8

Veljanoski, J., Mackey, A. D., Ferguson, A. M. N., et al. 2014, MNRAS, 442, 2929

Ventura, P., \& D’Antona, F. 2009, A\&A, 499, 835

Ventura, P., \& D’Antona, F. 2011, MNRAS, 410, 2760

Ventura, P., D’Antona, F., Mazzitelli, I., \& Gratton, R. 2001, ApJ, 550, L65

Ventura, P., Di Criscienzo, M., Carini, R., \& D’Antona, F. 2013, MNRAS, 431, 3642

Vesperini, E., McMillan, S. L. W., D’Antona, F., \& D’Ercole, A. 2010, ApJ, 718, L112

Villanova, S., Geisler, D., Carraro, G., Moni Bidin, C., \& Muñoz, C. 2013, ApJ, 778,186

von Glasow, W., Krause, M. G. H., Sommer-Larsen, J., \& Burkert, A. 2013, MNRAS, 434, 1151

Voss, R., Diehl, R., Hartmann, D. H., et al. 2009, A\&A, 504, 531

Voss, R., Diehl, R., Vink, J. S., \& Hartmann, D. H. 2010, A\&A, 520, A51

Voss, R., Martin, P., Diehl, R., et al. 2012, A\&A, 539, A66

Webb, J. J., Sills, A., Harris, W. E., \& Hurley, J. R. 2014, MNRAS, 445, 1048

Werchan, F., \& Zaritsky, D. 2011, AJ, 142, 48

Whitmore, B. C., Chandar, R., Kim, H., et al. 2011, ApJ, 729, 78

Wu, Z.-Y., Du, C.-H., Ma, J., \& Zhou, X. 2009, Chin. Phys. Lett., 26, 029701

Zinnecker, H., \& Yorke, H. W. 2007, ARA\&A, 45, 481

Zocchi, A., Bertin, G., \& Varri, A. L. 2012, A\&A, 539, A65 


\section{Appendix A: Gas pressure versus radiation pressure}

In our models we consider the effect of gas pressure alone because the radiation pressure is generally too low to lead to gas expulsion in massive clusters. We verified this by calculating the total luminosity of massive stars $\left(>8 M_{\odot}\right)$ for the low-metallicity $[\mathrm{Fe} / \mathrm{H}]=-1.5 \mathrm{FRMS}$ grid of Decressin et al. (2007) and for the model grids of moderately rotating stars at solar metallicity and $[\mathrm{Fe} / \mathrm{H}]=-0.8$ from Ekström et al. (2012) and Georgy et al. (2013), respectively. For example, a $M_{*}=10^{6} M_{\odot}$ young star cluster produces a total luminosity of $L=1 \times 10^{43} \mathrm{erg} \mathrm{s}^{-1}$ during the first few Myr of its lifetime this varies by a factor of two for the different model grids. For efficient scattering or absorption of the entire spectrum, the force exerted by this radiation on a shell would be $f_{1} f_{2} L / c$, where the factor $f_{1}$ is lower than unity and accounts for the fact that a part of the stellar distribution is outside the shell, and $f_{2}$ may exceed unity and accounts for multiple scatterings. The energy transferred to the shell after the latter would have been lifted to the half-mass radius $r_{\mathrm{h}}$ would then be $E_{\mathrm{R}}=F_{1} F_{2} L r_{\mathrm{h}} / c=3 \times 10^{51} F_{1} F_{2} r_{\mathrm{h}, 3}$ erg, where $F_{1}$ and $F_{2}$ now refer to path averages of the respective factors $f_{1}$ and $f_{2}$. This compares to the binding energy of the gas (Baumgardt et al. $2008), E_{\mathrm{G}}=0.4\left(1-\epsilon_{\mathrm{SF}}\right)\left(M_{*} / \epsilon_{\mathrm{SF}}\right)^{2} / r_{\mathrm{h}}=9 \times 10^{52} r_{\mathrm{h}, 3}^{-1}$, where we have used $\epsilon_{\mathrm{SF}}=0.3$ as an example. Numerical simulations of radiation-pressure-driven outflows find efficiency factors $f_{2}$ not significantly above unity (Krumholz \& Thompson 2013), which is due to instabilities that tend to create optically thin regions where the radiation escapes easily. Therefore, the energy delivered by the radiation force does not reach the binding energy for this example. Because the binding energy scales with $M_{*}^{2}$, while the energy produced by all stellar feedback processes including radiation pressure is linear in the mass, this statement is even stronger for higher masses.

By comparison, using even the low-metallicity $([\mathrm{Fe} / \mathrm{H}]=-1.5)$ wind powers from the stellar models of Decressin et al. (2007), the stellar winds need about 4.5 Myr to produce an energy equivalent to the binding energy for the above example. Taking an efficiency factor of 0.1 into account (compare Sect. 3.3), they still deliver $1.4 \times 10^{52}$ erg within the first 3.5 Myr, that is before the first supernova.

We note that the presence of hot gas in superbubbles has recently been disputed (Murray et al. 2010), based on low X-ray luminosity in some objects and a possible overprediction of the total X-ray luminosity of star-forming galaxies. However, 3D hydrodynamics simulations with time-dependent energy input have shown that, thanks to 3D instabilities, the X-ray luminosity is strongly variable on Myr timescales and that the integrated X-ray luminosity for star-forming galaxies is well below the observed values, leaving room for contributions from other X-ray sources (Krause et al. 2014).

Radiation pressure may therefore safely be neglected for the studies in this paper. It might also be thought of as being included in the stellar wind power with our adopted efficiency factor of 0.2 . 
M. G. H. Krause et al.: Gas expulsion in massive star clusters?

\section{Appendix B: Additional table}

Table B.1. Data for star clusters that have been searched for the Na-O anti-correlation or multiple populations in the colour-magnitude diagram.

\begin{tabular}{|c|c|c|c|c|c|c|c|c|c|}
\hline Class & Object & Alt. name & $\begin{array}{c}M_{*} \\
\left(10^{5} M_{\odot}\right)\end{array}$ & $\begin{array}{l}r_{\mathrm{h}}{ }^{a} \\
(\mathrm{pc})\end{array}$ & $C_{5}{ }^{b}$ & $\begin{array}{l}\text { Age } \\
\text { (Gyr) }\end{array}$ & {$[\mathrm{Fe} / \mathrm{H}]$} & $\mathrm{MPs}^{c}$ & Ref. \\
\hline $\mathrm{GC}$ & $\begin{array}{ll}\text { NGC } & 104\end{array}$ & 47 Tuc & 6.46 & 7.1 & 0.92 & 12.8 & -0.76 & $\bar{Y}$ & $1,2,3,4$ \\
\hline $\mathrm{GC}$ & NGC 288 & Melotte 3 & 0.46 & 9.8 & 0.05 & 12.2 & -1.32 & $\mathrm{Y}$ & $1,2,3,4$ \\
\hline $\mathrm{GC}$ & NGC 362 & Dunlop 62 & 2.5 & 3.5 & 0.72 & 10 & -1.26 & $\mathrm{Y}$ & $2,3,4,5$ \\
\hline $\mathrm{GC}$ & NGC 1261 & Caldwell 87 & 3.41 & 5.5 & 0.62 & 10.24 & -1.08 & $\mathrm{Y}$ & $2,4,6,7$ \\
\hline $\mathrm{GC}$ & NGC 1851 & Dunlop 508 & 5.51 & 3.05 & 1.81 & 7.64 & -1.13 & Y & $2,4,6,8$ \\
\hline $\mathrm{GC}$ & NGC 1904 & M79 & 1.45 & 4.1 & 0.50 & 12.0 & -1.58 & Y & $1,2,9$ \\
\hline $\mathrm{GC}$ & NGC 2298 & Dunlop 578 & 0.85 & 3.1 & 0.16 & 12.4 & -1.98 & Y & $2,4,6,8$ \\
\hline $\mathrm{GC}$ & NGC 2808 & Dunlop 265 & 12.3 & 3.8 & 3.24 & 11.2 & -1.18 & Y & $1,2,3,4$ \\
\hline GC & NGC 3201 & Dunlop 445 & 1.1 & 7.5 & 0.15 & 11.1 & -1.51 & Y & $1,2,4,10$ \\
\hline $\mathrm{GC}$ & NGC 4590 & M68 & 1.07 & 7.7 & 0.14 & 12.7 & -2.27 & $\mathrm{Y}$ & $1,2,3,4$ \\
\hline $\mathrm{GC}$ & NGC 4833 & Dunlop 164 & 4.10 & 7.9 & 0.52 & 12.5 & -1.71 & Y & $2,4,6,7$ \\
\hline $\mathrm{GC}$ & NGC 5024 & M53 & 8.26 & 11.6 & 0.71 & 12.7 & -1.86 & $\mathrm{Y}$ & $2,4,6,7$ \\
\hline $\mathrm{GC}$ & NGC 5053 & & 1.25 & 22.5 & 0.056 & 12.3 & -1.98 & Y & $2,4,6,7$ \\
\hline $\mathrm{GC}$ & NGC 5272 & M3 & 4.68 & 11.7 & 0.40 & 11.4 & -1.34 & Y & $2,3,4,7$ \\
\hline $\mathrm{GC}$ & NGC 5286 & & 7.13 & 4.2 & 1.69 & 12.5 & -1.41 & Y & $2,3,4,7$ \\
\hline GC & NGC 5466 & & 1.79 & 18.2 & 0.098 & 13.6 & -2.20 & Y & $2,4,6,7$ \\
\hline $\mathrm{GC}$ & NGC 5897 & & 2.11 & 12.7 & 0.17 & 12.3 & -1.73 & Y & $2,4,6,7$ \\
\hline $\mathrm{GC}$ & NGC 5904 & M5 & 3.89 & 6.6 & 0.59 & 11.5 & -1.33 & $\mathrm{Y}$ & $1,2,3,4$ \\
\hline $\mathrm{GC}$ & NGC 5927 & & 3.38 & 4.2 & 0.81 & 12.7 & -0.64 & Y & $2,4,6,7$ \\
\hline $\mathrm{GC}$ & NGC 5986 & & 5.99 & 5.04 & 1.19 & 12.2 & -1.35 & $\mathrm{Y}$ & $2,4,6,7$ \\
\hline GC & NGC 6093 & M80 & 5.02 & 3.02 & 1.66 & 12.5 & -1.47 & Y & $2,4,6,7$ \\
\hline GC & NGC 6101 & Caldwell 107 & 1.0 & 8.00 & 0.13 & 12.5 & -1.76 & Y & $2,4,7,11$ \\
\hline $\mathrm{GC}$ & NGC 6121 & M4 & 1.17 & 4.7 & 0.25 & 13.1 & -1.98 & Y & $1,2,3,4$ \\
\hline $\mathrm{GC}$ & NGC 6139 & & 3.80 & 4.2 & 0.90 & 11.6 & -1.58 & $\mathrm{Y}$ & $1,2,12$ \\
\hline $\mathrm{GC}$ & NGC 6144 & & 1.69 & 7.17 & 0.24 & 13.8 & -1.56 & $\mathrm{Y}$ & $2,4,6,7$ \\
\hline $\mathrm{GC}$ & NGC 6171 & M107 & 1.17 & 5.5 & 0.21 & 13.4 & -1.03 & $\mathrm{Y}$ & $1,2,4,13,14$ \\
\hline $\mathrm{GC}$ & NGC 6205 & M13 & 7.75 & 5.93 & 1.31 & 11.7 & -1.33 & $\mathrm{Y}$ & $2,4,6,7$ \\
\hline $\mathrm{GC}$ & NGC 6218 & M12 & 0.74 & 4.2 & 0.18 & 13.4 & -1.43 & Y & $1,2,3,4$ \\
\hline GC & NGC 6254 & M10 & 1.53 & 4.2 & 0.36 & 12.4 & -1.57 & Y & $1,2,4,15$ \\
\hline $\mathrm{GC}$ & NGC 6304 & & 2.17 & 4.14 & 0.52 & 13.6 & -0.66 & Y & $2,4,6,7$ \\
\hline GC & NGC 6341 & M92 & 4.89 & 4.19 & 1.17 & 13.2 & -2.16 & $\mathrm{Y}$ & $2,4,6,7$ \\
\hline $\mathrm{GC}$ & NGC 6352 & & 0.37 & 5.68 & 0.065 & 12.7 & -0.70 & Y & $2,4,7,16$ \\
\hline $\mathrm{GC}$ & NGC 6362 & & 0.81 & 7.70 & 0.11 & 13.6 & -0.99 & Y & $2,4,7,16$ \\
\hline $\mathrm{GC}$ & NGC 6366 & & 0.30 & 5.05 & 0.058 & 13.3 & -0.73 & Y & $2,4,7,16$ \\
\hline $\mathrm{GC}$ & NGC 6388 & & 12 & 2.5 & 4.8 & 11.7 & -0.45 & Y & $1,2,4,17$ \\
\hline GC & NGC 6397 & & 1.1 & 3.3 & 0.33 & 13.4 & -1.99 & Y & $1,2,4,18$ \\
\hline $\mathrm{GC}$ & NGC 6441 & & 9.55 & 3.3 & 2.92 & 11.2 & -0.44 & Y & $1,2,3,4$ \\
\hline GC & NGC 6496 & & 2.00 & 5.70 & 0.35 & 12.4 & -0.70 & Y & $2,4,6,7$ \\
\hline $\mathrm{GC}$ & NGC 6535 & & 0.20 & 2.86 & 0.070 & 10.5 & -1.51 & Y & $2,4,6,7$ \\
\hline $\mathrm{GC}$ & NGC 6541 & & 5.72 & 3.93 & 1.45 & 12.9 & -1.53 & Y & $2,4,6,7$ \\
\hline $\mathrm{GC}$ & NGC 6584 & & 3.03 & 4.87 & 0.62 & 11.3 & -1.30 & Y & $2,4,6,7$ \\
\hline GC & NGC 6624 & & 2.57 & 3.20 & 0.80 & 12.5 & -0.70 & Y & $2,4,6,7$ \\
\hline GC & NGC 6637 & M69 & 2.00 & 3.66 & 0.55 & 13.1 & -0.78 & Y & $2,4,7,19$ \\
\hline GC & NGC 6652 & & 1.09 & 2.37 & 0.46 & 12.0 & -0.97 & Y & $2,4,6,7$ \\
\hline
\end{tabular}

Notes. ${ }^{(a)}$ Half-mass radii were computed from the half-light radii (apparent size was taken as equivalent to half-light radii) from the literature, assuming a Plummer model for the cluster. ${ }^{(b)}$ Compactness $M_{*} / r_{\mathrm{h}}$ in units of $10^{5} M_{\odot} / \mathrm{pc}$. ${ }^{(c)}$ Y: possesses multiple populations; N: multiple populations have been searched for, but not found. ${ }^{(d)}$ Estimated from Fig. 5 in Ref. (16).

References. (1) Carretta et al. (2010); (2) Harris (1996, 2010 edition); (3) Kimmig et al. (2015); (4) Piotto et al. (2015): (5) Lebzelter \& Wood (2011); (6) Boyles et al. (2011); (7) Forbes \& Bridges (2010); (8) Koleva et al. (2008); (9) Lützgendorf et al. (2013); (10) Mucciarelli et al. (2015); (11) Caloi \& D'Antona (2011); (12) Bragaglia et al. (2015) (13) Conroy (2012); (14) Piatek et al. (1994); (15) Zocchi et al. (2012) (16) Mandushev et al. (1991); (17) Lanzoni et al. (2013); (18) Heyl et al. (2012); (19) Miocchi (2007) (20) Villanova et al. (2013); (21) Cantat-Gaudin et al. (2014); (22) Salaris \& Weiss (2002); (23) Bragaglia et al. (2012); (24) Tadross (2001) (25) Bragaglia \& Tosi (2006) (26) Mikolaitis et al. (2012); (27) Casewell et al. (2014); (28) Lodieu et al. (2014); (29) MacLean et al. (2015); (30) Portegies Zwart et al. (2001) (31) Gebran et al. (2008) (32) Becker \& Fenkart (1971); (33) Francic (1989); (34) Reddy et al. (2012); (35) Donati et al. (2014); (36) Gozha et al. (2012); (37) Güneş et al. (2012); (38) Lee et al. (2013); (39) Marconi et al. (1997); (40) Hurley et al. (2005); (41) Pancino et al. (2010); (42) Santrich et al. (2013); (43) Smiljanic et al. (2009) (44) Mikolaitis et al. (2010) (45) Bragaglia et al. (2014) (46) Platais et al. (2011); (47) Wu et al. (2009) (48) Mikolaitis et al. (2011); (49) Larsen et al. (2012); (50) Goudfrooij et al. (2014); (51) Mucciarelli et al. (2008); (52) Niederhofer et al. in prep; (53) Mackey \& Gilmore (2003) (54) Mucciarelli et al. (2009); (55) Mucciarelli et al. (2010) (56) Bastian \& Niederhofer (2015); (57) Mucciarelli et al. (2014); (58) Mackey et al. (in prep.); (59) Bastian \& Silva-Villa (2013); (60) Mucciarelli et al. (2011); (61) Niederhofer et al. (2015); (62) Ferraro et al. (2006); (63) Milone et al. (2009); (64) Mucciarelli et al. (2007); (65) Werchan \& Zaritsky (2011); (66) Davies et al. (2009); (67) Portegies Zwart et al. (2010). 
Table B.1. continued.

\begin{tabular}{|c|c|c|c|c|c|c|c|c|c|}
\hline Class & Object & Alt. name & $\begin{array}{c}M_{*} \\
\left(10^{5} M_{\odot}\right)\end{array}$ & $\begin{array}{l}r_{\mathrm{h}}{ }^{a} \\
(\mathrm{pc})\end{array}$ & $C_{5}^{b}$ & $\begin{array}{l}\text { Age } \\
\text { (Gyr) }\end{array}$ & {$[\mathrm{Fe} / \mathrm{H}]$} & $\mathrm{MPs}^{c}$ & Ref. \\
\hline $\mathrm{GC}$ & NGC 6656 & M22 & 6.44 & 5.32 & 1.21 & 12.7 & -1.49 & $\mathrm{Y}$ & $2,4,6,7$ \\
\hline GC & NGC 6681 & M70 & 1.79 & 3.16 & 0.57 & 12.8 & -1.35 & $\mathrm{Y}$ & $2,4,6,7$ \\
\hline GC & NGC 6715 & M54 & 12.9 & 10.7 & 1.20 & 10.8 & -1.25 & $\mathrm{Y}$ & $2,3,4,7$ \\
\hline GC & NGC 6717 & & 0.48 & 2.39 & 0.20 & 13.2 & -1.09 & $\mathrm{Y}$ & $2,4,6,7$ \\
\hline GC & NGC 6723 & & 3.57 & 6.58 & 0.54 & 13.1 & -0.96 & $\mathrm{Y}$ & $2,4,6,7$ \\
\hline GC & NGC 6752 & & 2.82 & 3.8 & 0.75 & 13.8 & -1.55 & $\mathrm{Y}$ & $1,2,3,4$ \\
\hline GC & NGC 6779 & M56 & 2.30 & 5.11 & 0.45 & 13.7 & -2.00 & $\mathrm{Y}$ & $2,4,6,7$ \\
\hline GC & NGC 6809 & M55 & 0.55 & 7.6 & 0.07 & 13.8 & -1.93 & $\mathrm{Y}$ & $1,2,3,4$ \\
\hline GC & NGC 6838 & M71 & 0.20 & 3.3 & 0.06 & 12.7 & -0.82 & Y & $1,2,3,4$ \\
\hline GC & NGC 6934 & & 2.95 & 5.32 & 0.55 & 11.1 & -1.32 & Y & $2,4,6,7$ \\
\hline GC & NGC 6981 & M72 & 1.68 & 7.82 & 0.21 & 10.9 & -1.21 & Y & $2,4,6,7$ \\
\hline GC & NGC 7078 & M15 & 5.13 & 5.1 & 1.00 & 13.6 & -2.33 & Y & $1,2,3,4$ \\
\hline GC & NGC 7089 & M2 & 5.75 & 6.03 & 0.95 & 11.8 & -1.31 & Y & $2,3,4,7$ \\
\hline GC & NGC 7099 & M30 & 1.45 & 4.1 & 0.35 & 14.6 & -2.33 & Y & $1,2,3,4$ \\
\hline GC & Ruprecht 106 & & 0.68 & 11 & 0.06 & 12 & -1.5 & $\mathbf{N}$ & 2,20 \\
\hline GC & Terzan 7 & & 0.39 & 8.7 & 0.04 & 7.4 & -0.12 & $\mathrm{~N}$ & $1,2,21,22$ \\
\hline GC & Palomar 12 & & 0.28 & 16 & 0.024 & 8.6 & -0.81 & $\mathrm{~N}$ & $1,2,21$ \\
\hline $\mathrm{OC}$ & Berkley 39 & & 0.2 & 11 & 0.018 & 6 & -0.21 & $\mathrm{~N}$ & 23,24 \\
\hline $\mathrm{OC}$ & Collinder 261 & & 0.01 & $2^{d}$ & 0.005 & 6 & -0.03 & $\mathrm{~N}$ & $24,25,26$ \\
\hline $\mathrm{OC}$ & Melotte 25 & Hyades & 0.004 & 3.7 & 0.001 & 0.6 & +0.13 & $\mathrm{~N}$ & $27,28,29,30$ \\
\hline $\mathrm{OC}$ & Melotte 111 & Coma Berenices & 0.001 & 3.5 & 0.0003 & 0.5 & +0.07 & $\mathrm{~N}$ & $27,29,31,32$ \\
\hline $\mathrm{OC}$ & NGC 752 & & 0.001 & 1.4 & 0.0009 & 1.12 & -0.02 & $\mathrm{~N}$ & $29,33,34$ \\
\hline $\mathrm{OC}$ & NGC 1817 & Collinder $4 \mathrm{a}$ & 0.02 & 7 & 0.002 & 1 & -0.11 & $\mathrm{~N}$ & $27,32,34,35,36$ \\
\hline $\mathrm{OC}$ & NGC 2360 & Melotte 64 & 0.018 & 2 & 0.009 & 0.56 & -0.07 & $\mathrm{~N}$ & $29,34,37$ \\
\hline $\mathrm{OC}$ & NGC 2506 & Collinder 170 & 0.03 & 15 & 0.002 & 1.11 & -0.19 & $\mathrm{~N}$ & $29,34,38,39$ \\
\hline $\mathrm{OC}$ & NGC 2682 & M67 & 0.01 & 2.5 & 0.004 & 4.4 & +0.05 & $\mathrm{~N}$ & $29,40,41$ \\
\hline $\mathrm{OC}$ & NGC 3114 & & 0.0016 & 8 & 0.0002 & 0.16 & -0.01 & $\mathrm{~N}$ & $24,29,36,42$ \\
\hline $\mathrm{OC}$ & NGC 6134 & & 0.0008 & 2 & 0.0004 & 0.7 & +0.12 & $\mathrm{~N}$ & $24,29,43,44$ \\
\hline $\mathrm{OC}$ & NGC 6253 & Melotte 156 & 0.007 & 2 & 0.0035 & 4 & +0.4 & $\mathrm{~N}$ & $24,26,29$ \\
\hline $\mathrm{OC}$ & NGC 6475 & M 7 & 0.007 & 6 & 0.001 & 0.3 & +0.14 & $\mathrm{~N}$ & $29,32,36$ \\
\hline $\mathrm{OC}$ & NGC 6705 & & 0.1 & 1.2 & 0.08 & 0.3 & +0.10 & $\mathrm{~N}$ & 21 \\
\hline $\mathrm{OC}$ & NGC 6791 & Berkeley 46 & 0.5 & 5 & 0.1 & 7.5 & +0.30 & $\mathrm{~N}$ & $29,45,46$ \\
\hline $\mathrm{OC}$ & NGC 7789 & Melotte 245 & 0.06 & 5 & 0.01 & 1.6 & +0.04 & $\mathrm{~N}$ & $29,36,41,47$ \\
\hline $\mathrm{OC}$ & IC 4651 & Melotte 169 & 0.006 & 2 & 0.003 & 1.1 & +0.15 & $\mathrm{~N}$ & $24,29,36,48$ \\
\hline Fornax & For 3 & & 5.2 & 7.2 & 0.72 & 13 & -2.33 & $\mathrm{Y}$ & 49 \\
\hline Fornax & For 5 & & 3.2 & 8.5 & 0.38 & 13 & -2.09 & $\mathrm{Y}$ & 49 \\
\hline LMC & NGC 1651 & & 0.8 & 22 & 0.04 & 1.5 & -0.3 & $\mathrm{~N}$ & $50,51,52$ \\
\hline LMC & NGC 1783 & & 2.5 & 19 & 0.13 & 1.4 & -0.3 & $\mathrm{~N}$ & $50,51,52$ \\
\hline LMC & NGC 1786 & & 3.7 & 6.0 & 0.62 & 13 & -1.75 & $\mathrm{Y}$ & $53,54,55$ \\
\hline LMC & NGC 1806 & & 1.3 & 15 & 0.08 & 1.4 & -0.3 & $\mathrm{~N}$ & $50,56,57$ \\
\hline LMC & NGC 1846 & & 1.7 & 15 & 0.11 & 1.4 & -0.3 & $\mathrm{~N}$ & $50,56,58$ \\
\hline LMC & NGC 1866 & & 0.8 & 19 & 0.04 & 0.2 & -0.3 & $\mathrm{~N}$ & $50,59,60,61$ \\
\hline LMC & NGC 1978 & & 2.0 & 8.7 & 0.23 & 2.0 & -0.3 & $\mathrm{~N}$ & $50,51,62,63,64,65$ \\
\hline LMC & NGC 2173 & & 0.5 & 11 & 0.05 & 1.6 & -0.3 & $\mathrm{~N}$ & $50,51,52$ \\
\hline LMC & NGC 2210 & & 3.0 & 5.9 & 0.51 & 13 & -1.65 & $\mathrm{Y}$ & $53,54,55$ \\
\hline LMC & NGC 2257 & & 2.6 & 19 & 0.14 & 13 & -1.95 & $\mathrm{Y}$ & $53,54,55$ \\
\hline \multicolumn{3}{|c|}{ Scutum red supergiant cluster 1} & 0.32 & 2.6 & 0.12 & 0.012 & -0.15 & $\mathrm{~N}$ & 66,67 \\
\hline \multicolumn{3}{|c|}{ Scutum red supergiant cluster 2} & 0.40 & 4.6 & 0.087 & 0.017 & -0.15 & $\mathrm{~N}$ & 66,67 \\
\hline
\end{tabular}

